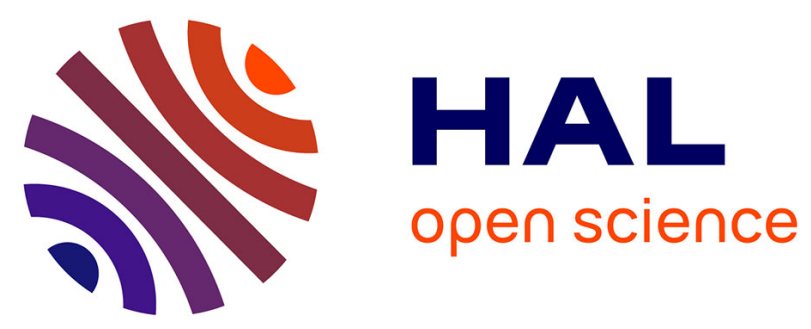

\title{
ON THE BOLTZMANN GAS MIXTURE EQUATION: LINKING THE KINETIC AND FLUID REGIMES
}

\author{
Carlo Bianca, Christian Dogbe
}

\section{To cite this version:}

Carlo Bianca, Christian Dogbe. ON THE BOLTZMANN GAS MIXTURE EQUATION: LINKING THE KINETIC AND FLUID REGIMES. Communications in Nonlinear Science and Numerical Simulation, 2015, 10.1016/j.cnsns.2015.05.015 . hal-02151785

\section{HAL Id: hal-02151785 \\ https://hal.science/hal-02151785}

Submitted on 10 Jun 2019

HAL is a multi-disciplinary open access archive for the deposit and dissemination of scientific research documents, whether they are published or not. The documents may come from teaching and research institutions in France or abroad, or from public or private research centers.
L'archive ouverte pluridisciplinaire HAL, est destinée au dépôt et à la diffusion de documents scientifiques de niveau recherche, publiés ou non, émanant des établissements d'enseignement et de recherche français ou étrangers, des laboratoires publics ou privés. 


\title{
ON THE BOLTZMANN GAS MIXTURE EQUATION: LINKING THE KINETIC AND FLUID REGIMES
}

\author{
CARLO BIANCA AND CHRISTIAN DOGBE
}

\begin{abstract}
This paper aims at developing a new connection between the Boltzmann equation and the Navier-Stokes equation. Specifically the paper deals with the derivation of the macroscopic equations from asymptotic limits of the Boltzmann equation for a binary gas mixture of hard-sphere gases. By extending the methodology of the singlecomponent gases case and by employing different time and space scalings, we show that it is possible to recover, under suitable technical assumptions, various fluid dynamics equations like the incompressible linearized and nonlinear Navier-Stokes equations, the incompressible linearized and nonlinear Euler equations. The novelty of this paper is the method that we propose, which differs from the Hilbert and Chapman-Enskog expansions. Future research directions are also discussed in the last section of the paper with special attention at the different scalings that can be employed in order to obtain equations presenting a ghost effect.
\end{abstract}

Keywords: Kinetic theory of gases; Boltzmann equation; gas mixtures; rarefied gas dynamics., hydrodynamics limits.

AMS Subject Classification (2010): 82C40, 76P05, 35Q35

\section{INTRODUCTION}

The statistical description of a rarefied gas, but in general of a thermodynamic system not in thermodynamic equilibrium, dates back to Ludwig Boltzmann when in 1872 proposed his celebrated equation. Since the derivation of the classical Boltzmann equation various issues have remained elusive but a very substantial progress has been made in the study of kinetic models for a gas composed of a very large number of identical particles, moving in a three-dimensional space. In this context the interest in the statistical description of a gas mixture has gained much attention and the theory for a simple gas has been generalized for gas mixtures, see the paper of Sirovich and Thurber [29].

A gas mixture is a physical system more complex than a single fluid, even if one considers non-reactive (inert) mixtures. To the best of our knowledge, the first investigation on the dynamics of a gas mixture goes back to Fick [20] in 1855. Subsequently the derivation of the Boltzmann equation for binary gas mixtures has been devised, see [15]. Recently, Kosuge et al. [28] have investigated the case of a binary mixture of hard-sphere gases confined between two parallel plates for a large difference of temperature. The interested reader in a more deeper understanding of this topic is referred to, among others, papers [1], [2], [16].

The present paper deals with the mathematical derivation of incompressible fluid mechanics equations, such as the Navier-Stokes (or Euler) equations, from the Boltzmann equation for binary inert gas mixture. The paper thus aims at developing a new connection between the Boltzmann equation and the Navier-Stokes equation. Specifically the paper deals with the derivation of the macroscopic equations for a binary gas mixture of hard-sphere gases from asymptotic limits of the Boltzmann equation. By extending the methodology of the single-component gases case proposed in [17] and by employing different time and space scalings, we show that it is possible to recover, under suitable technical assumptions, various fluid dynamics equations like the incompressible linearized and nonlinear Navier-Stokes 
equations, the incompressible linearized and nonlinear Euler equations. It is also worth mentioning the papers $[3,4,18]$ where a general class of kinetic models with collisional integral having the classical collision invariants is considered and whose asymptotic limit leads to the classical equations of the incompressible fluid mechanics.

Differently from the single-component gases case, the pertinent literature for the gaseous mixtures case is more limited. In $[32,36]$ the condensation-vaporization problem for the mixture of vapors of different species has been considered. The derivation of incompressible Navier-Stokes equations from Boltzmann equations that model the dynamics of gases whose particles may undergo nonelastic collisions has been proposed in [9]. In [11], starting from a kinetic model for a chemical reaction, the authors have derived multi-temperature reactive Euler equations. In $[23,25,37]$ the authors have considered the Vlasov-Boltzmann system for a fluid of two species of interacting particles and have performed diffusive expansions, in terms of Knudsen numbers, for the solutions of the rescaled system around Maxwellians of equal weights for the two species. The formal passage from a kinetic model to the incompressible Navier-Stokes equations for a mixture of monoatomic gases with different masses has been performed in [10]. In [34] the authors deals with an interesting proof of an almost exponential decay rate of solutions near the Maxwellian equilibrium. It is worth stressing that in the multispecies Boltzmann equation, each species does not conserve the momentum and energy, although these quantities are conserved for the entire systems, making difficult the hydrodynamic limit when, for example, the characteristic microscopic time is of the order of the relaxation time of the temperature among the species [26].

The novelty of this paper is the method that we propose for deriving the hydrodynamic equations from the Boltzmann equation, which differs from the Hilbert and Chapman-Enskog expansions. Specifically the method consists in passing to the limit as the Knudsen number vanishes in the local conservation laws of mass, momentum and energy that are satisfied by "well-behaved" solutions of the Boltzmann gas mixture equations system.

It is worth stressing that a general strategy for deriving global hydrodynamic limits leading to incompressible models was proposed by Bardos, Golse and Levermore [4, 5, 3] who have considered the hydrodynamic limit problems related to single species cases by considering as underlying framework the Chapman and Enskog framework [15] with more general collision kernels. In particular in $[4,5,3]$ the authors have shown that the compressible Navier-Stokes equations is the governing hydrodynamic equations if the first order approximation is included when the Mach number is a constant and the Knudsen number goes to zero, and the hydrodynamic equation is the incompressible Navier-Stokes equations when the Mach number goes to zero with the same rate as the Knudsen number. A different approach has been recently proposed for the asymptotic limit of generalized Boltzmann-type equations in which the action of a thermostat has been taken into account, see [7], [8].

The contents of this paper are organized as follows. After this introduction, Section 2 deals with the underlying framework, which is the binary gaseous mixture Boltzmann equation, and we collect some basic ingredients and known results that we will use later into the paper. In Section 3 we consider various scalings which lead to the derivation of the hydrodynamic models by introducing the dimensionless parameter in the problem: the scaled interaction mean-free path $\varepsilon$. The scalings that are of interest are the hyperbolic scaling and the parabolic scaling. The proof of the main result is given in Section 4. Finally Section 5 is concerned with further research directions on the possibility to derive macroscopic equations by employing generalized scaling with the aim to obtain the limiting ghost effect system.

\section{The Binary Gaseous Mixture Boltzmann Equation}

This section is concerned with the presentation of the underlying framework that will be subjected to the asymptotic analysis. Specifically we consider the evolution of a binary 
gaseous mixture, e.g. the mixture of the $L$-component and the $H$-component. In what follows, the Greek letters $\alpha, \ell$ are symbolically used to represent the two species having masses $m^{\alpha}$ and $m^{\ell}$, that is say $\{\alpha, \ell\} \in\{L, H\}$.

Let $F^{\alpha}=F^{\alpha}(t, x, v)$ be the distribution function of the $\alpha$-component that is solution of the following binary gaseous mixture Boltzmann equations system (see Kogan [27], Chapman and Cowling [15], Ferziger and Kaper [19], Hirschfelder et al. [24]):

$$
\begin{aligned}
\partial_{t} F^{\mathrm{L}}+v \cdot \nabla_{x} F^{\mathrm{L}} & =\frac{2}{\sqrt{\pi}} \frac{1}{\mathrm{Kn}}\left(Q\left(F^{\mathrm{L}}, F^{\mathrm{L}}\right)+Q\left(F^{\mathrm{L}}, F^{\mathrm{H}}\right)\right) \\
\partial_{t} F^{\mathrm{H}}+v \cdot \nabla_{x} F^{\mathrm{H}} & =\frac{2}{\sqrt{\pi}} \frac{1}{\mathrm{Kn}}\left(Q\left(F^{\mathrm{H}}, F^{\mathrm{L}}\right)+Q\left(F^{\mathrm{H}}, F^{\mathrm{H}}\right)\right)
\end{aligned}
$$

where $\mathrm{Kn}$ is the Knudsen number and $Q\left(F^{\alpha}, F^{\ell}\right)=Q\left(F^{\alpha}, F^{\ell}\right)(t, x, v)$ is the Boltzmann collision operator:

$$
\begin{array}{r}
Q\left(F^{\alpha}, F^{\ell}\right)=\int_{\mathbb{S}^{+} \times \mathbb{R}^{3}}\left[F^{\alpha}\left(v_{*}^{\prime}\right) F^{\ell}\left(v^{\prime}\right)-F^{\alpha}\left(v_{*}\right) F^{\ell}(v)\right] B^{\ell \alpha}\left(\left|v_{*}-v\right|, \omega\right) d \omega d v_{*}, \\
F\left(v_{*}^{\prime}\right)=F\left(t, x, v_{*}^{\prime}\right), \quad F\left(v^{\prime}\right)=F\left(t, x, v^{\prime}\right), \quad F\left(v_{*}\right)=F\left(t, x, v_{*}\right), \quad F(v)=F(t, x, v),
\end{array}
$$

where $\mathbb{S}^{+}$is the half-sphere defined by $\left(v-v_{*}\right) \cdot \omega$, with $\omega$ an unit vector, $\mathbf{v}:=v_{*}-v$ is the relative velocity, $d \omega$ is the solid angle element in the direction of $\omega, B^{\ell \alpha}\left(=B^{\alpha \ell}\right)$ is a nonnegative function, whose functional form is determined by the intermolecular force between species $\ell$ and $\alpha$. In particular $B^{\ell \alpha}$ is the collision kernel that corresponds to the hard-sphere gas case (cross-section). The integration in Eq. (2.2) is carried out over the whole space of $v_{*}$ and the whole direction of $\omega$. The domain of integration appearing in the sequel is, unless otherwise stated, the whole space of the integration variables. Since we are not dealing with free particles, at least because of collisions, under some physical assumptions, the Boltzmann collision operator, is an operator acting only w.r.t. velocity variable $v$.

Assuming elastic collisions among the particles, the post-collisional velocities $v^{\prime}$ and $v_{*}^{\prime}$ reads:

$$
v^{\prime}=v+\frac{2 m^{\ell}}{m^{\alpha}+m^{\ell}}(\omega \cdot \mathbf{v}) \omega, \quad v_{*}^{\prime}=v_{*}-\frac{2 m^{\alpha}}{m^{\alpha}+m^{\ell}}(\omega \cdot \mathbf{v}) \omega
$$

Relation (2.3) can be obtained from momentum and energy conservation during the collision $\left(v, v_{*}\right) \leftrightarrow\left(v^{\prime}, v_{*}^{\prime}\right)$, that read:

$$
\begin{aligned}
& m^{\alpha} v^{\prime}+m^{\ell} v_{*}^{\prime}=m^{\alpha} v+m^{\ell} v_{*} \\
& m^{\alpha}\left|v^{\prime}\right|^{2}+m^{\ell}\left|v_{*}^{\prime}\right|^{2}=m^{\alpha}|v|^{2}+m^{\ell}\left|v_{*}\right|^{2}
\end{aligned}
$$

where $m^{\alpha}$ is the molecular mass of the $\alpha$-component.

As already mentioned, in this paper we consider the following hard-sphere form for the function $B^{\ell \alpha}$ appearing in the collision term (2.2) (see [15]):

$$
B^{\ell \alpha}=\frac{\left(d^{\ell \alpha}\right)^{2}}{2}|\omega \cdot V|, \quad d^{\ell \alpha}=\frac{d^{\ell}+d^{\alpha}}{2}
$$

being $d^{\alpha}$ the molecular diameter of species $\alpha$.

It is worth stressing that if the right-hand side of (2.1) is set to zero, then this equation would describe the free behavior of particles; one then obtains a transport equation.

The main aim of this paper is to investigate the steady behavior for small values of the Knudsen number $\mathrm{Kn}$, especially in the continuum limit where $\mathrm{Kn}$ vanishes. We underline that there is no external force in the system. 
2.1. Properties of the collision operators. Denoting $F=\left(F^{L}, F^{\mathrm{H}}\right)^{T}$ (in which " $T$ " means vector transpose), the collision operator can be written in compact form as follows:

$$
\mathscr{C}(F):=\left(\begin{array}{c}
Q\left(F^{L}, F^{L}\right)+Q\left(F^{\mathrm{H}}, F^{L}\right) \\
Q\left(F^{L}, F^{\mathrm{H}}\right)+Q\left(F^{\mathrm{H}}, F^{\mathrm{H}}\right)
\end{array}\right)
$$

and consequently the "two-species" Boltzmann equation now reads:

$$
\partial_{t} F+v \cdot \nabla_{x} F=\mathscr{C}(F) .
$$

In order to clarify the collisions between two types of particles we use the following notations:

$$
Q^{L} \equiv Q\left(F^{\mathrm{L}}, F^{\mathrm{L}}\right), \quad Q^{\mathrm{LH}} \equiv Q\left(F^{\mathrm{L}}, F^{\mathrm{H}}\right), \quad Q^{\mathrm{HL}} \equiv Q\left(F^{\mathrm{H}}, F^{\mathrm{L}}\right), \quad Q^{\mathrm{H}} \equiv Q\left(F^{\mathrm{H}}, F^{\mathrm{H}}\right) .
$$

Setting

$$
\psi \equiv m^{\mathrm{L}} F^{\mathrm{L}}+m^{\mathrm{H}} F^{\mathrm{H}} \quad \text { and } \quad G \equiv m^{\mathrm{L}} F^{\mathrm{L}}-m^{\mathrm{H}} F^{\mathrm{H}},
$$

the system (2.1) can be rewritten as follows:

$$
\begin{gathered}
\partial_{t} \psi+v \cdot \nabla_{x} \psi=m^{\mathrm{L}}\left(Q^{\mathrm{L}}+Q^{\mathrm{LH}}\right)+m^{\mathrm{H}}\left(Q^{\mathrm{HL}}+Q^{\mathrm{H}}\right) \\
\partial_{t} G+v \cdot \nabla_{x} G=m^{\mathrm{L}}\left(Q^{\mathrm{L}}+Q^{\mathrm{LH}}\right)-m^{\mathrm{H}}\left(Q^{\mathrm{HL}}+Q^{\mathrm{H}}\right) .
\end{gathered}
$$

We remark that, when $m^{\mathrm{L}}=m^{\mathrm{H}}=m$, i.e. the particles have unit mass then, the right-handsides of (2.7) can be significantly simplified: ${ }^{1}$

$$
\begin{array}{r}
\partial_{t} \psi+v \cdot \nabla_{x} \psi=Q(\psi, \psi) \\
\partial_{t} G+v \cdot \nabla_{x} G=Q(G, G) .
\end{array}
$$

In what follows we summarize the main properties of the collision operator $Q$ that are fundamental in the study of the mixture equation (for details see, for instance the articles by $[22],[21],[31],[2],[17])$ :

(i) Mass conservation:

$$
\int_{\mathbb{R}^{3}} Q^{L} d v=0, \quad \int_{\mathbb{R}^{3}} Q^{\mathrm{LH}} d v=0, \quad \int_{\mathbb{R}^{3}} Q^{\mathrm{HL}} d v=0, \quad \int_{\mathbb{R}^{3}} Q^{\mathrm{H}} d v=0,
$$

(ii) Momentum conservation:

$$
\int_{\mathbb{R}^{3}} Q^{L} m^{\mathrm{L}} v d v=0, \quad \int_{\mathbb{R}^{3}}\left(Q^{\mathrm{LH}} m^{\mathrm{L}} v+Q^{\mathrm{HL}} m^{\mathrm{H}} v\right) d v=0, \quad \int_{\mathbb{R}^{3}} Q^{\mathrm{H}} m^{\mathrm{H}} v d v=0,
$$

(iii) Energy conservation:

$$
\int_{\mathbb{R}^{3}} Q^{L} m^{\mathrm{L}}|v|^{2} d v=0, \quad \int_{\mathbb{R}^{3}}\left(Q^{\mathrm{LH}} m^{\mathrm{L}}|v|^{2}+Q^{\mathrm{HL}} m^{\mathrm{H}}|v|^{2}\right) d v=0, \quad \int_{\mathbb{R}^{3}} Q^{\mathrm{H}} m^{\mathrm{H}}|v|^{2} d v=0,
$$

(iv) Entropy inequalities: For any function $G \in \mathscr{C}_{0}^{\infty}\left(\mathbb{R}^{+} \times \mathbb{R}_{v}^{3}\right)$ and for any $G=\left(G^{\mathrm{L}}, G^{\mathrm{H}}\right)^{T}$, the following inequality holds:

$$
(\mathscr{C}(G), \log G)_{\left(L^{2}\left(\mathbb{R}^{3}\right)\right)^{2}}=\left(\mathscr{C}(G),\left(\begin{array}{l}
\log G^{L} \\
\log G^{\mathrm{H}}
\end{array}\right)\right)_{\left(L^{2}\left(\mathbb{R}^{3}\right)\right)^{2}} \leq 0 .
$$

\footnotetext{
${ }^{1}$ It turns out that it is convenient to consider the sum and difference of $F^{\mathrm{L}}$ and $F^{\mathrm{H}}$, as proposed in [6]
} 
(v) Local thermal equilibria: As a consequence of the entropy inequality, any equilibrium distribution, namely any distribution which maximizes the entropy, has the form of a locally Maxwellian distribution. That is, for any $G=\left(G^{\mathrm{L}}, G^{\mathrm{H}}\right)^{T}, \quad(\mathscr{C}(G), \log G)=$ $0 \Leftrightarrow G$ "is bi-Maxwellian":

$$
G=\left(\begin{array}{l}
n^{\mathrm{L}}\left(\frac{m^{\mathrm{L}}}{2 \pi \theta}\right)^{3 / 2} \exp \left(-m^{\mathrm{L}} \frac{|v-u|^{2}}{2 \theta}\right) \\
n^{\mathrm{H}}\left(\frac{m^{\mathrm{H}}}{2 \pi \theta}\right)^{3 / 2} \exp \left(-m^{\mathrm{H}} \frac{|v-u|^{2}}{2 \theta}\right)
\end{array}\right),
$$

where $u^{\mathrm{L}}=u^{\mathrm{H}}=u \in \mathbb{R}^{3}, \theta^{\mathrm{L}}=\theta^{\mathrm{H}}=\theta \in \mathbb{R}^{+}$for any $L$ and $H$ and $n^{\alpha}=\int_{\mathbb{R}^{3}} G^{\alpha} d v \in \mathbb{R}^{+}$ is the number density of gas $\alpha$; that is the two Maxwellians have the same local temperature and mean velocities, with $n^{\alpha}>0$ to maintain the positivity of the distribution function and $\theta>0$ to guarantee its integrability with respect to $v$. Here $(\cdot, \cdot)$ denotes the inner product in $\left(L^{2}\left(\mathbb{R}^{3}\right)\right)^{2}$.

For simplicity we set:

$$
\begin{aligned}
\mathcal{M}^{\mathrm{L}} & \equiv \mathcal{M}_{\left[n^{\mathrm{L}}, u, \theta\right]}(v)=n^{\mathrm{L}}\left(\frac{m^{\mathrm{L}}}{2 \pi \theta}\right)^{3 / 2} \exp \left(-m^{\mathrm{L}} \frac{|v-u|^{2}}{2 \theta}\right) \\
\mathcal{M}^{\mathrm{H}} & \equiv \mathcal{M}_{\left[n^{\mathrm{H}}, u, \theta\right]}(v)=n^{\mathrm{H}}\left(\frac{m^{\mathrm{H}}}{2 \pi \theta}\right)^{3 / 2} \exp \left(-m^{\mathrm{H}} \frac{|v-u|^{2}}{2 \theta}\right) .
\end{aligned}
$$

The density $n^{\alpha}$, the mean velocity $u^{\alpha}=u$ and the temperature $\theta^{\alpha}=\theta$ are such that

$$
\int \mathcal{M}_{\left[n^{\alpha}, u, \theta\right]}(v)\left(\begin{array}{c}
1 \\
m^{\alpha} v \\
m^{\alpha}|v|^{2}
\end{array}\right) d v=\left(\begin{array}{c}
n^{\alpha} \\
m^{\alpha} n^{\alpha} u \\
m^{\alpha} n^{\alpha}|u|^{2}+3 \theta
\end{array}\right) .
$$

The quantities $m^{\alpha} n^{\alpha} u^{\alpha}$ and $\mathcal{W}_{\mathcal{M}}^{\alpha}:=\frac{1}{2} m^{\alpha} n^{\alpha}|u|^{2}+\frac{3}{2} \theta$ are the momentum and energy densities of the Maxwellian. We have the same property for the species $H$.

When $u=0$, we define

$$
\mu^{\mathrm{L}}(v)=n^{\mathrm{L}}\left(\frac{m^{\mathrm{L}}}{2 \pi \theta}\right)^{3 / 2} \exp \left(-m^{\mathrm{L}} \frac{|v|^{2}}{2 \theta}\right), \quad \mu^{\mathrm{H}}(v)=n^{\mathrm{H}}\left(\frac{m^{\mathrm{H}}}{2 \pi \theta}\right)^{3 / 2} \exp \left(-m^{\mathrm{H}} \frac{|v|^{2}}{2 \theta}\right) .
$$

It is worth noting that if we define the scaling transformations of the functions defined on $\mathbb{R}^{3}$ by

we get

$$
\Lambda_{u} f(\xi)=f(\xi-u), \quad m_{\lambda} f(\xi)=\lambda^{-3} f\left(\frac{\xi}{\lambda}\right)
$$

$$
\mathcal{M}_{(n, u, \theta)}(v)=n m_{\sqrt{\theta}} \Lambda_{u} \mathcal{M}_{(1,0,1)} .
$$

Hence it is enough to study the linearization of the collision integral at the Gaussian,

$$
\mathcal{M}_{(1,0,1)}(v)=\frac{1}{(2 \pi)^{3 / 2}} e^{-\frac{|v|^{2}}{2}}
$$

which greatly simplifies the calculations involved.

Bearing all above in mind, we thus define $M_{(1,0,1)}^{\alpha}$ as an absolute normalized Maxwellians with number density equal to 1 , mass velocity equal to 0 , temperature equal to 1 , i.e.

$$
\mathcal{M}^{\alpha} \equiv \mathcal{M}_{[1,0,1]}(v)(v)=\left(\frac{m^{\alpha}}{2 \pi}\right)^{3 / 2} \exp \left(-m^{\alpha} \frac{|v|^{2}}{2}\right) \text {. }
$$


2.2. Moments and conservation laws. Macroscopic quantities such as the number, momentum and energy densities can be constructed from integrals of the distribution function with respect to the velocity. We call such quantities "moments". The density $n^{\alpha}$, mean velocity $u^{\alpha}$ and the energy density $\mathcal{W}^{\alpha}$ of the species $\alpha$ can be computed from the $\alpha$-species distribution function $f^{\alpha}$ according to

$$
\left(\begin{array}{c}
n^{\alpha} \\
m^{\alpha} n^{\alpha} u^{\alpha} \\
\mathcal{W}^{\alpha}
\end{array}\right)=\int F^{\alpha}(v)\left(\begin{array}{c}
1 \\
m^{\alpha} v^{\alpha} \\
\frac{1}{2} m^{\alpha}|v|^{2}
\end{array}\right) d v
$$

The quantity $m^{\alpha} n^{\alpha} u^{\alpha}$ is the $\alpha$-species momentum density and $\mathcal{W}^{\alpha}$ is the energy density. Moreover the following fields can be defined:

$$
\begin{array}{ll}
\mathbf{c}=v-u^{\alpha} & (\text { random velocity with bulk velocity } u) \\
q_{i}^{\alpha}=\frac{1}{2} \int_{\mathbb{R}^{3}} \mathbf{c}_{i}|c|^{2} F^{\alpha} d v & (i=1,2), \quad(\text { heat flow vector) } \\
p_{i j}^{\alpha}=\int_{\mathbb{R}^{3}} \mathbf{c}_{i} \mathbf{c}_{j} F^{\alpha} d v \quad(i, j=1,2), \quad\left(\text { stress tensor with components } p_{i j}^{\alpha}\right) .
\end{array}
$$

It is common to separate the drift motion (defined by the average velocity $u^{\alpha}$ ) and the random kinetic motion (defined by the velocity $\mathbf{c}$ ) in evaluating these integrals. By definition of $u^{\alpha}$, one has

where

$$
\int v \otimes v F^{\alpha} m^{\alpha} d v=m^{\alpha} n^{\alpha} u^{\alpha} \otimes u^{\alpha}+\mathbb{P}^{\alpha}
$$

and

$$
\mathbb{P}^{\alpha}:=\operatorname{trace}\left(p_{i j}\right)=\int\left(v-u^{\alpha}\right) \otimes\left(v-u^{\alpha}\right) m^{\alpha} F^{\alpha} d v, \quad(i, j=1,2),
$$

with

$$
\int \frac{1}{2} m^{\alpha}|v|^{2} v F^{\alpha} m^{\alpha} d v=\mathcal{W}^{\alpha} u^{\alpha}+\mathbb{P}^{\alpha} u^{\alpha}+\mathbb{Q}^{\alpha}
$$

$$
\mathbb{Q}^{\alpha}:=\int \frac{m^{\alpha}\left|v-u^{\alpha}\right|^{2}}{2}\left(v-u^{\alpha}\right) F^{\alpha} d v
$$

The internal energy per particle $e^{\alpha}$ and the temperature $\theta^{\alpha}$ of $\alpha$-species gas are defined as:

$$
e^{\alpha}=\frac{m^{\alpha}}{2 n^{\alpha}} \int_{\mathbb{R}^{3}}\left|v-u^{\alpha}\right|^{2} F^{\alpha} d v
$$

where, and in the sequel unless otherwise stated, the domain of integration is the whole space of $v$ (or of the variable of integration). We define the mass density $\varrho^{\alpha}$ by $\varrho^{\alpha}=m^{\alpha} n^{\alpha}$. We also define global quantities for the mixture: the counterparts of the mixture, i.e., the molecular number density $n$, mass density $\varrho$, mass average velocity $v$, pressure $p$ and temperature $\theta$, are expressed by a proper combination of the quantities above as

$$
n=\sum_{\alpha \in\{L, H\}} n^{\alpha}, \quad \varrho=\sum_{\alpha \in\{L, H\}} \varrho^{\alpha}, \quad E=n \theta=\sum_{\alpha \in\{L, H\}}\left(p^{\alpha}+\frac{1}{3}\left|u-v^{\alpha}\right|^{2}\right) .
$$

The following proposition holds true, see [17] for the proof.

Proposition 2.1. Let $F^{\alpha}=F^{\alpha}(t, x, v)$ be a solution of the Boltzmann mixture system (2.1) that is locally integrable and rapidly decaying in $v$ for each $(t, x)$. Then the following local 
conservation laws hold:

$$
\begin{gathered}
\partial_{t} \int_{\mathbb{R}^{3}} F^{\alpha} v d v+\nabla_{x} \int_{\mathbb{R}^{3}} v F^{\alpha} d v=0 \\
\partial_{t} \int_{\mathbb{R}^{3}} m^{\alpha} v F^{\alpha} d v+\nabla_{x} \int_{\mathbb{R}^{3}} m^{\alpha} v \otimes v F^{\alpha} d v=\int_{\mathbb{R}^{3}} m^{\alpha} v Q\left(F^{\alpha}, F^{\ell}\right) d v \\
\partial_{t} \int_{\mathbb{R}^{3}} m^{\alpha} \frac{1}{2}|v|^{2} F^{\alpha} d v+\nabla_{x} \int_{\mathbb{R}^{3}} m^{\alpha} \frac{1}{2}|v| L^{2} v F^{\alpha} d v=\int_{\mathbb{R}^{3}} m^{\alpha} \frac{1}{2}|v|^{2} Q\left(F^{\alpha}, F^{\ell}\right) d v
\end{gathered}
$$

respectively the local conservation of mass, momentum and energy.

Bearing the previous notations for the thermodynamic fields in mind, these continuity equations are, for the $L$-species:

$$
\begin{gathered}
\partial_{t} n^{\mathrm{L}}+\nabla_{x} \cdot\left(n^{\mathrm{L}} u\right)=0 \\
\partial_{t}\left(m^{\mathrm{L}} n^{\mathrm{L}} u\right)+\nabla_{x}\left(\int v \otimes v F^{\mathrm{L}} m^{\mathrm{L}} d v\right)=\mathcal{Q}^{\mathrm{LH}} \\
\partial_{t} \mathcal{W}^{\mathrm{L}}+\nabla_{x} \cdot\left(\int \frac{m^{\mathrm{L}}|v|^{2}}{2} v F^{\mathrm{L}} d v\right)=\mathscr{Q}^{\mathrm{LH}}
\end{gathered}
$$

and similarly for the species $H$ :

$$
\begin{gathered}
\partial_{t} n^{\mathrm{H}}+\nabla_{x} \cdot\left(n^{\mathrm{H}} u\right)=0 \\
\partial_{t}\left(m^{\mathrm{H}} n^{\mathrm{H}} u\right)+\nabla_{x}\left(\int v \otimes v F^{\mathrm{H}} m^{\mathrm{H}} d v\right)=\mathcal{Q}^{\mathrm{HL}} \\
\partial_{t} \mathcal{W}^{\mathrm{H}}+\nabla_{x} \cdot\left(\int \frac{m^{\mathrm{H}}|v|^{2}}{2} v F^{\mathrm{H}} d v\right)=\mathscr{Q}^{\mathrm{HL}},
\end{gathered}
$$

where $\mathcal{Q}^{\alpha \ell}$ and $\mathscr{Q}^{\alpha \ell}$ are the momentum and energy transfer rates toward species $\alpha$ from species $\ell$ :

$$
\left(\begin{array}{c}
\mathcal{Q}^{\alpha \ell} \\
\mathscr{Q}^{\ell \alpha}
\end{array}\right)=\int Q^{\alpha \ell}\left(F^{\alpha}, F^{\ell}\right)(v)\left(\begin{array}{c}
m^{\alpha} v \\
\frac{1}{2} m^{\alpha}|v|^{2}
\end{array}\right) d v
$$

so that, because of the momentum and energy conservation properties of the unlike-collision operators (2.10) and (2.11), we have

$$
\mathcal{Q}^{\alpha \ell}+\mathcal{Q}^{\ell \alpha}=0, \quad \mathscr{Q}^{\alpha \ell}+\mathscr{Q}^{\ell \alpha}=0 .
$$

2.3. Linearization near global Maxwellians. In this subsection, we recall briefly some basic ingredients on the linearized Boltzmann equation for a binary gaseous mixture.

Firstly we simplify the far field in (2.14). We further normalize the collision operator $(2.2)$ as

$$
Q\left(F^{\alpha}, F^{\ell}\right)=\int_{\mathbb{S}^{+} \times \mathbb{R}^{3}}\left|\left(v_{*}-v\right), \omega\right|\left[F^{\alpha}\left(v_{*}^{\prime}\right) F^{\ell}\left(v^{\prime}\right)-F^{\alpha}\left(v_{*}\right) F^{\ell}(v)\right] d \omega d v_{*} .
$$

Our main concern will be to study the operator (2.25) "close" to the equilibrium position. We will reformulate the problem (2.1) as a perturbation of the equilibria. More explicitly, if we define

$$
F^{\alpha}=\mu_{\alpha}+\sqrt{\mu_{\alpha}} f_{\alpha}, \quad \alpha \in\{\mathrm{L}, \mathrm{H}\} .
$$

as the standard perturbation of $F^{\alpha}(t, x, v)$ to $\sqrt{\mu_{\alpha}}$, we observe that the quadratic collision operator (2.2) satisfies

$$
Q^{\mathrm{L}}\left(\mathcal{M}^{\mathrm{L}}, \mathcal{M}^{\mathrm{L}}\right)+Q^{\mathrm{LH}}\left(\mathcal{M}^{\mathrm{L}}, \mathcal{M}^{\mathrm{H}}\right)=0, \quad \text { and } \quad Q^{\mathrm{HL}}\left(\mathcal{M}^{\mathrm{H}}, \mathcal{M}^{\mathrm{L}}\right)+Q^{\mathrm{H}}\left(\mathcal{M}^{\mathrm{H}}, \mathcal{M}^{\mathrm{H}}\right)=0 .
$$

Therefore, the linearized collision operator $\mathcal{L} g$, for $g=\left[g^{\mathrm{L}}, g^{\mathrm{H}}\right]$, is defined by

$$
\mathcal{L} g=[\mathbb{L} g, \mathbb{H} g], \quad \mathbb{L} g \equiv-\mathcal{A}^{\mathrm{L}} g-\mathcal{K}^{\mathrm{L}} g, \quad \mathbb{H} g \equiv-\mathcal{A}^{\mathrm{H}} g-\mathcal{K}^{\mathrm{H}} g
$$


where

$$
\begin{aligned}
\mathcal{A}^{\mathrm{L}} g & \equiv \mu_{\mathrm{L}}^{-1 / 2} Q\left(\sqrt{\mu_{\mathrm{L}}} g^{\mathrm{L}}, \mu_{\mathrm{L}}\right)+\mu_{\mathrm{L}}^{-1 / 2} Q\left(\sqrt{\mu_{\mathrm{L}}} g^{\mathrm{L}}, \mu_{\mathrm{H}}\right) \\
\mathcal{A}^{\mathrm{H}} g & \equiv \mu_{\mathrm{H}}^{-1 / 2} Q\left(\sqrt{\mu_{\mathrm{H}}} g^{\mathrm{H}}, \mu_{\mathrm{H}}\right)+\mu_{\mathrm{H}}^{-1 / 2} Q\left(\sqrt{\mu_{\mathrm{H}}} g^{\mathrm{H}}, \mu_{\mathrm{L}}\right) \\
\mathcal{K}^{\mathrm{L}} g & \equiv \mu_{\mathrm{L}}^{-1 / 2} Q\left(\mu_{\mathrm{L}}, \sqrt{\mu_{\mathrm{L}}} g^{\mathrm{L}}\right)+\mu_{\mathrm{L}}^{-1 / 2} Q\left(\mu_{\mathrm{L}}, \sqrt{\mu_{\mathrm{H}}} g^{\mathrm{H}}\right) \\
\mathcal{K}^{\mathrm{H}} g & \equiv \mu_{\mathrm{H}}^{-1 / 2} Q\left(\mu_{\mathrm{H}}, \sqrt{\mu_{\mathrm{H}}} g^{\mathrm{H}}\right)+\mu_{\mathrm{H}}^{-1 / 2} Q\left(\mu_{\mathrm{H}}, \sqrt{\mu_{\mathrm{L}}} g^{\mathrm{L}}\right),
\end{aligned}
$$

and the nonlinear part of the collision operator (2.2) is defined by

$$
\begin{gathered}
\Gamma^{\mathrm{L}}(g, h)=\mu_{\mathrm{L}}^{-1 / 2} Q\left(\sqrt{\mu_{\mathrm{L}}} g^{\mathrm{L}}, \sqrt{\mu_{\mathrm{L}}} h^{\mathrm{L}}\right)+\mu_{\mathrm{L}}^{-1 / 2} Q\left(\sqrt{\mu_{\mathrm{L}}} g^{\mathrm{L}}, \sqrt{\mu_{\mathrm{H}}} h^{\mathrm{H}}\right) \\
\Gamma^{\mathrm{H}}(g, h)=\mu_{\mathrm{H}}^{-1 / 2} Q\left(\sqrt{\mu_{\mathrm{H}}} g^{\mathrm{H}}, \sqrt{\mu_{\mathrm{H}}} h^{\mathrm{H}}\right)+\mu_{\mathrm{H}}^{-1 / 2} Q\left(\sqrt{\mu_{\mathrm{H}}} g^{\mathrm{H}}, \sqrt{\mu_{\mathrm{L}}} h^{\mathrm{L}}\right) .
\end{gathered}
$$

As expected from the H-theorem, $\mathcal{L}$ is non-negative and for every fixed $(t, x)$ the null space of $\mathcal{L}$ is given by the six dimensional space.

We denote by $L_{\mathcal{M}}^{2}$ the associated Hilbert space, $\langle\cdot, \cdot\rangle$ denotes the usual $L^{2}$ inner product without the weight. We summarize the properties of $\mathcal{L}$ in the following lemma (see Proposition 2.1, pp. 635-638, [1]):

Lemma 2.2. We assume the hard-sphere interaction for the collision kernel.

1. $\mathcal{L}$ is the sum of a diagonal operator $f \mapsto \mathcal{A} f$

$$
\mathcal{A} f=\left(\begin{array}{l}
\mathcal{A}^{L}(v) f^{L} \\
\nu^{H}(v) f^{H}
\end{array}\right), \quad \text { with } \mathcal{A}^{\alpha}(|v|) \sim 1+|v|
$$

and a compact operator $\mathcal{K}$. The domain of $\mathcal{L}$ is given by

$$
\mathcal{D}(\mathcal{L})=\left\{f:\left\|(1+|v|)^{\frac{1}{2}} f\right\|_{L_{\mathcal{M}}^{1}}<\infty\right\} .
$$

2. $\mathcal{L}$ is self-adjoint in $L_{\mathcal{M}}^{2}$ :

$$
\left\langle\mathcal{L}\left(\begin{array}{l}
f^{L} \\
f^{H}
\end{array}\right),\left(\begin{array}{l}
g^{L} \\
g^{H}
\end{array}\right)\right\rangle_{\mathcal{M}}=\left\langle\left(\begin{array}{l}
f^{L} \\
f^{H}
\end{array}\right), \mathcal{L}\left(\begin{array}{l}
g^{L} \\
g^{H}
\end{array}\right)\right\rangle_{\mathcal{M}}
$$

$\mathcal{L}$ is nonnegative.

3. The kernel of $\mathcal{L}$ is a six-dimensional linear space:

where

$$
\operatorname{ker}(\mathcal{L})=\operatorname{Span}\left\{\phi_{i}, \quad i=0, \ldots, 5\right\}
$$

$$
\phi_{0}=\left(\begin{array}{l}
1 \\
0
\end{array}\right), \phi_{1}=\left(\begin{array}{l}
0 \\
1
\end{array}\right), \phi_{i+1}=\left(\begin{array}{c}
m^{L} v_{i} \\
m^{H} v_{i}
\end{array}\right),(i=1,2,3), \quad \phi_{5}=\left(\begin{array}{c}
m^{L}|v|^{2} \\
m^{H}|v|^{2}
\end{array}\right) .
$$

4. Any function $f \in \mathcal{D}(\mathcal{L})$ can be written as $f=q_{f}+w_{f}$ with $q_{f} \in \operatorname{ker}(\mathcal{L})$ and $w \in(\operatorname{ker}(\mathcal{L}))^{\perp}$ and we have $\langle\mathbb{L} f, f\rangle_{\mathcal{M}} \geqslant \delta_{0}\left\|(1+|v|)^{\frac{1}{2}} w_{f}\right\|^{2}$.

\section{Linking the Kinetic and Fluid Regimes: Multiscale Analysis}

This section aims at showing how to connect the kinetic and fluid regimes via the method of asymptotic expansions. The derivations (2.21) and (2.22) can be rigorous, if we take a sequence of solutions $F_{\varepsilon}^{\alpha}, \alpha \in\{\mathrm{L}, \mathrm{H}\}$ of (2.1), where the Knudsen number $\varepsilon:=\frac{\sqrt{\pi}}{2} \mathrm{Kn}$ goes to 0 . The Knudsen number $\mathrm{Kn}$ is a small parameter defined by the ratio $\ell_{0} / L$ where $\ell_{0}$ is the mean free path and $L$ is the typical length of the system. 
3.1. The rescaling problem. Different macroscopic models correspond to different scaling assumptions. The so-called parabolic (low field) limit of kinetic equations leads to a driftdiffusion type system (or reaction-diffusion system) in which the diffusion processes dominate the behavior of the solutions. On the other hand, in the hyperbolic (high field) limit the influence of the diffusion terms is of lower (or equal) order of magnitude in comparison with other convective or interaction terms and the aim is the derivation of hyperbolic macroscopic models.

Since all the collisions are completely elastic, we have the following scattering cross sections: $\sigma^{\mathrm{L}}$ (self collision for two L species); $\sigma^{\mathrm{H}}$ (self collision for two $\mathrm{H}$ species); $\sigma^{\mathrm{LH}}$ (cross collision for $\mathrm{H}$ specie and $\mathrm{L}$ specie). We define:

$$
\nu_{\mathrm{HH}}=\frac{\sigma^{\mathrm{H}}}{m^{\mathrm{H}}}, \quad \nu_{\mathrm{HL}}=\frac{\sigma^{\mathrm{HL}}}{m^{\mathrm{H}}} \quad \nu_{\mathrm{LL}}=\frac{\sigma^{\mathrm{L}}}{m^{\mathrm{L}}}, \quad \nu_{\mathrm{LH}}=\frac{\sigma^{\mathrm{LH}}}{m^{\mathrm{L}}}
$$

where, $\nu_{\mathrm{LL}}$ and $\nu_{\mathrm{HH}}$ are the frequencies of self-collisions, $\nu_{\mathrm{LH}}$ is frequency of collisions of $\mathrm{L}$ molecules with $\mathrm{H}$ molecules, and $\nu_{\mathrm{HL}}$ is frequency of collisions of $\mathrm{H}$ molecules with $\mathrm{L}$ molecules. In order to take into account the collision between the species, we rewrite (2.1) as

$$
\begin{aligned}
\partial_{t} F^{\mathrm{H}}+v \cdot \nabla_{x} F^{\mathrm{H}} & =\nu_{\mathrm{HL}} Q\left(F^{\mathrm{H}}, F^{\mathrm{L}}\right)+\nu_{\mathrm{HH}} Q\left(F^{\mathrm{H}}, F^{\mathrm{H}}\right) \\
\partial_{t} F^{\mathrm{L}}+v \cdot \nabla_{x} F^{\mathrm{L}} & =\nu_{\mathrm{LL}} Q\left(F^{\mathrm{L}}, F^{\mathrm{L}}\right)+\nu_{\mathrm{LH}} Q^{\mathrm{LH}}\left(F^{\mathrm{L}}, F^{\mathrm{H}}\right) .
\end{aligned}
$$

We will look at the solution of (3.2a) at time $\varepsilon^{-1} t$ and space $\varepsilon^{-1} x$ (hyperbolic scaling or Euler scaling) by setting

$$
F_{\varepsilon}^{\mathrm{H}}(t, x, v)=F^{\mathrm{H}}\left(\varepsilon^{-1} t, \varepsilon^{-1} x, v\right)
$$

together with the following choice of the frequency of collisions:

$$
\nu_{\mathrm{HL}}=\varepsilon^{q}, \quad q>1, \quad \nu_{\mathrm{HH}}=O(1) .
$$

We will look at the solution of (3.2b) at a time $\varepsilon^{-2} t$ and space $\varepsilon^{-1} x$ (diffusion scaling) by setting

$$
F_{\varepsilon}^{\mathrm{L}}(t, x, v)=F^{\mathrm{L}}\left(\varepsilon^{-2} t, \varepsilon^{-1} x, v\right)
$$

together with the following choice of the frequency of collisions:

$$
\nu_{\mathrm{LH}}=\varepsilon^{q+1}, \quad q>1, \quad \nu_{\mathrm{LL}}=O(1) .
$$

The rescaled distributions $F_{\varepsilon}^{\mathrm{H}}(t, x, v)$ and $F_{\varepsilon}^{\mathrm{L}}(t, x, v)$ have to solve the rescaled equation:

$$
\begin{aligned}
\partial_{t} F_{\varepsilon}^{\mathrm{H}}+v \cdot \nabla_{x} F_{\varepsilon}^{\mathrm{H}} & =\varepsilon^{q} Q\left(F_{\varepsilon}^{\mathrm{H}}, F_{\varepsilon}^{\mathrm{L}}\right)+\frac{1}{\varepsilon} Q\left(F_{\varepsilon}^{\mathrm{H}}, F_{\varepsilon}^{\mathrm{H}}\right) \\
\varepsilon \partial_{t} F_{\varepsilon}^{\mathrm{L}}+v \cdot \nabla_{x} F_{\varepsilon}^{\mathrm{L}} & =\frac{1}{\varepsilon} Q\left(F_{\varepsilon}^{\mathrm{L}}, F_{\varepsilon}^{\mathrm{L}}\right)+\varepsilon^{q+1} Q\left(F_{\varepsilon}^{\mathrm{L}}, F_{\varepsilon}^{\mathrm{H}}\right) .
\end{aligned}
$$

We are looking for the diffusion/hydrodynamic asymptotic limit as $\varepsilon \rightarrow 0$. Clearly, Maxwelization for the $L$-species occurs faster than for the $H$-species, which is consistent with the physical evidence. This choice is mainly guided by the structure of the collision operator. We introduce the scaled time variable defined by

$$
t=\frac{t^{\prime}}{\varepsilon^{\gamma}}
$$

where $\gamma \geqslant 0$ is the strength parameter of the scaling; this time scaling which is related to the Strouhal number (ratio of the oscillation frequency to the bulk velocity) is introduced to suppress, when $\gamma \neq 0$, the acoustic modes varying in a faster timescale than rotational modes of the fluid. The Boltzmann mixture equations (3.7a)-(3.7b) become

$$
\begin{aligned}
\varepsilon^{\gamma} \partial_{t} F_{\varepsilon}^{\mathrm{H}}+v \cdot \nabla_{x} F_{\varepsilon}^{\mathrm{H}} & =\varepsilon^{q} Q\left(F_{\varepsilon}^{\mathrm{H}}, F_{\varepsilon}^{\mathrm{L}}\right)+\frac{1}{\varepsilon} Q\left(F_{\varepsilon}^{\mathrm{H}}, F_{\varepsilon}^{\mathrm{H}}\right) \\
\varepsilon^{\gamma+1} \partial_{t} F_{\varepsilon}^{\mathrm{L}}+v \cdot \nabla_{x} F_{\varepsilon}^{\mathrm{L}} & =\frac{1}{\varepsilon} Q\left(F_{\varepsilon}^{\mathrm{L}}, F_{\varepsilon}^{\mathrm{L}}\right)+\varepsilon^{q+1} Q\left(F_{\varepsilon}^{\mathrm{L}}, F_{\varepsilon}^{\mathrm{H}}\right) .
\end{aligned}
$$


We expect to recover the hydrodynamical equations in the limit $\varepsilon \rightarrow 0$. Formally, it is clear that in this limit the right-hand side of (3.9a) would become singular. The only possibility to avoid this singularity is that

$$
\lim _{\varepsilon \rightarrow 0} Q\left(F_{\varepsilon}^{\mathrm{H}}, F_{\varepsilon}^{\mathrm{H}}\right)=0 .
$$

Then, by (2.13), the limit must be a Maxwellian, namely,

$$
F_{0}^{\mathrm{H}}=\mathcal{M}\left[n^{\mathrm{H}}, u, \theta\right],
$$

for some $\left(n^{\mathrm{H}}, u, \theta\right)$ which can be function of $t$ and $x$ and similarly for the specie $\mathrm{L}$.

First, if $F_{\varepsilon}^{\mathrm{L}}$ and $F_{\varepsilon}^{\mathrm{H}}$ solve respectively the binary gas mixture Boltzmann equations $(3.9 \mathrm{~b})$ and (3.9a) then $F_{\varepsilon}^{\mathrm{L}}$ satisfies the local conservation laws of mass, momentum, and energy:

$$
\begin{gathered}
\varepsilon^{\gamma+1} \partial_{t} \int_{\mathbb{R}^{3}} F_{\varepsilon}^{\mathrm{L}} d v+\nabla_{x} \cdot \int_{\mathbb{R}^{3}} v F_{\varepsilon}^{\mathrm{L}} d v=0, \\
\varepsilon^{\gamma+1} \partial_{t} \int_{\mathbb{R}^{3}} v m^{\mathrm{L}} F_{\varepsilon}^{\mathrm{L}} d v+\nabla_{x} \cdot \int_{\mathbb{R}^{3}} v \otimes v m^{\mathrm{L}} F_{\varepsilon}^{\mathrm{L}} d v=\varepsilon^{q+1} m^{\mathrm{L}} \mathcal{Q}\left(F_{\varepsilon}^{\mathrm{L}}, F_{\varepsilon}^{\mathrm{H}}\right), \\
\varepsilon^{\gamma+1} \partial_{t} \int_{\mathbb{R}^{3}} \frac{1}{2}|v|^{2} m^{\mathrm{L}} F_{\varepsilon}^{\mathrm{L}} d v+\nabla_{x} \cdot \int_{\mathbb{R}^{3}} v \frac{1}{2}|v|^{2} m^{\mathrm{L}} F_{\varepsilon}^{\mathrm{L}} d v=\varepsilon^{q+1} m^{\mathrm{L}} \mathscr{Q}\left(F_{\varepsilon}^{\mathrm{L}}, F_{\varepsilon}^{\mathrm{H}}\right),
\end{gathered}
$$

and for $F_{\varepsilon}^{\mathrm{H}}$ one has:

$$
\begin{gathered}
\varepsilon^{\gamma} \partial_{t} \int_{\mathbb{R}^{3}} F_{\varepsilon}^{\mathrm{H}} d v+\nabla_{x} \cdot \int_{\mathbb{R}^{3}} v F_{\varepsilon}^{\mathrm{H}} d v=0, \\
\varepsilon^{\gamma} \partial_{t} \int_{\mathbb{R}^{3}} v m^{\mathrm{H}} F_{\varepsilon}^{\mathrm{H}} d v+\nabla_{x} \cdot \int_{\mathbb{R}^{3}} v \otimes v m^{\mathrm{H}} F_{\varepsilon}^{\mathrm{H}} d v=\varepsilon^{q} m^{\mathrm{H}} \mathcal{Q}\left(F_{\varepsilon}^{\mathrm{H}}, F_{\varepsilon}^{\mathrm{L}}\right), \\
\varepsilon^{\gamma} \partial_{t} \int_{\mathbb{R}^{3}} \frac{1}{2}|v|^{2} m^{\mathrm{H}} F_{\varepsilon}^{\mathrm{H}} d v+\nabla_{x} \cdot \int_{\mathbb{R}^{3}} v \frac{1}{2}|v|^{2} m^{\mathrm{H}} F_{\varepsilon}^{\mathrm{H}} d v=\varepsilon^{q} m^{\mathrm{H}} \mathscr{Q}\left(F_{\varepsilon}^{\mathrm{H}}, F_{\varepsilon}^{\mathrm{L}}\right) .
\end{gathered}
$$

Since the solutions of the asymptotic equations are not guaranteed to exist or to be regular, our proof is only formal. We assume that for each $\varepsilon>0, F_{\varepsilon}^{\alpha}$ is a solution of (3.9a) and (3.9b) that satisfies the local conservation laws of mass, momentum, and energy, as well as the local entropy relation. Assume that

as well as

$$
F_{\varepsilon}^{\alpha} \rightarrow F^{\alpha} \text { a.e }
$$

$$
\begin{aligned}
\int_{\mathbb{R}^{3}} F_{\varepsilon}^{\alpha} d v & \rightarrow \int_{\mathbb{R}^{3}} F^{\alpha} d v & \text { in } \mathscr{C}\left(\mathbb{R}^{+} ; \mathscr{D}^{\prime}\left(\mathbb{R}^{3}\right)\right), \\
\int_{\mathbb{R}^{3}} v F_{\varepsilon}^{\alpha} d v & \rightarrow \int_{\mathbb{R}^{3}} v F^{\alpha} d v & \text { in } \mathscr{C}\left(\mathbb{R}^{+} ; \mathscr{D}^{\prime}\left(\mathbb{R}^{3}\right)\right), \\
\int_{\mathbb{R}^{3}}|v|^{2} F_{\varepsilon}^{\alpha} d v & \rightarrow \int_{\mathbb{R}^{3}}|v|^{2} F^{\alpha} d v & \text { in } \mathscr{C}\left(\mathbb{R}^{+} ; \mathscr{D}^{\prime}\left(\mathbb{R}^{3}\right)\right),
\end{aligned}
$$

while

$$
\begin{array}{lr}
\int_{\mathbb{R}^{3}} v \otimes v F_{\varepsilon}^{\alpha} d v \rightarrow \int_{\mathbb{R}^{3}} v \otimes v F^{\alpha} d v & \text { in } \mathscr{C}\left(\mathbb{R}^{+} ; \mathscr{D}^{\prime}\left(\mathbb{R}^{3}\right)\right), \\
\int_{\mathbb{R}^{3}} v|v|^{2} F_{\varepsilon}^{\alpha} d v \rightarrow \int_{\mathbb{R}^{3}} v|v|^{2} F^{\alpha} d v & \text { in } \mathscr{C}\left(\mathbb{R}^{+} ; \mathscr{D}^{\prime}\left(\mathbb{R}^{3}\right)\right),
\end{array}
$$

as $\varepsilon \rightarrow 0$. 
3.2. The Hydrodynamic Regime. We start from Boltzmann gas mixture system of equations (3.9b)-(3.9a). As shown by Bardos et al. [4] starting from the nonlinear Boltzmann equation, a whole family of scaled kinetic equations could be considered in general, with different scales in time, space and departure of the initial data from $\mu$ corresponding to different powers of $\varepsilon$. At the formal level of description it is then possible to recover, under suitable hypothesis, various fluid limits like the incompressible linearized and nonlinear Navier-Stokes equations, the incompressible linearized and nonlinear Euler equations.

The small Mach number (ratio of the bulk velocity to the sound speed) is realized if $F_{\varepsilon}^{\alpha}$ is close to an absolute Maxwellian. If one takes the standard Maxwellian, the distance to this absolute Maxwellian can be scaled in the unit of the Knudsen number $\varepsilon$ as

$$
F_{\varepsilon}^{\alpha}(t, x, v)=\mu_{\alpha}+\varepsilon^{\beta} \mu_{\alpha}^{1 / 2}(v) g_{\varepsilon}^{\alpha}\left(t^{\prime}, x, v\right), \quad \alpha \in\{L, H\}
$$

where $\beta \geqslant 0$ is the strength parameter of the scaling. The distribution function $g^{\alpha}$ can be viewed as the microscopic response of the system to gradients of macroscropic variables.

Varying the strengths of these two scalings yields different limits as $\varepsilon \rightarrow 0$. One can derive different fluid equations (and in particular incompressible models) depending on the chosen scaling.

It is worth stressing that we did not try to get all possible scalings. In particular, one can easily derive simplified models to those we have here.

Before going further into the analysis, a linearization of $F_{\varepsilon}^{\alpha}$ is required.

3.3. Solution near absolute Maxwellian. We will look for the solution $F_{\varepsilon}^{\alpha}$ near $\mu$, that is, the solution having the form

$$
F_{\varepsilon}^{\mathrm{H}}=\mu_{\mathrm{H}}+\varepsilon^{\beta} \mu_{\mathrm{H}}^{\frac{1}{2}} g_{\varepsilon}^{\mathrm{H}}, \quad F_{\varepsilon}^{\mathrm{L}}=\mu_{\mathrm{L}}+\varepsilon^{\mathbf{m}} \mu_{\mathrm{L}}^{\frac{1}{2}} g_{\varepsilon}^{\mathrm{L}},
$$

with $g_{\varepsilon}^{\alpha}=O(1)$ as $\varepsilon \rightarrow 0$.

Plugging the scaling (3.15) into the mixture system (3.9) to deduce the governing equation of the new unknown $g_{\varepsilon}^{\alpha}$, one finds:

$$
\begin{aligned}
\epsilon^{\gamma+1} \partial_{t} g_{\epsilon}^{L}+v \cdot \nabla_{x} g_{\epsilon}^{L}= & \frac{1}{\varepsilon} \mathbb{L}\left(g_{\epsilon}^{L}\right)+\varepsilon^{\mathbf{m}-\mathbf{1}} \Gamma\left(g_{\epsilon}^{L}\right)+\left(\mu_{\mathrm{L}}\right)^{-1 / 2}\left\{\epsilon^{\beta+q-m+1} Q^{L H}\left(\mu_{L}, \mu_{H}^{1 / 2} g_{\epsilon}^{H}\right)\right. \\
& \left.+\epsilon^{q+1} Q^{L H}\left(\mu_{L}^{1 / 2} g_{\epsilon}^{L}, \mu_{H}\right)+\epsilon^{\beta+q+1} Q^{L H}\left(\mu_{\mathrm{L}}^{\frac{1}{2}} g_{\varepsilon}^{\mathrm{L}}, \mu_{\mathrm{H}}^{\frac{1}{2}} g_{\varepsilon}^{\mathrm{H}}\right)\right\} \\
\varepsilon^{\gamma} \partial_{t} F_{\varepsilon}^{\mathrm{H}}+v \cdot \nabla_{x} F_{\varepsilon}^{\mathrm{H}}= & \frac{1}{\varepsilon} \mathbb{H}\left(g_{\epsilon}^{H}\right)+\varepsilon^{\beta-1} \Gamma\left(g_{\epsilon}^{H}\right)+\left(\mu_{\mathrm{H}}\right)^{-1 / 2}\left\{\varepsilon^{\mathbf{m}+\mathbf{q}-\beta} Q^{H L}\left(\mu_{H}, \mu_{\mathrm{L}}^{\frac{1}{2}} g_{\varepsilon}^{\mathrm{L}}\right)\right. \\
& \left.+\varepsilon^{\mathbf{q}} Q^{L H}\left(\mu_{\mathrm{H}}^{\frac{1}{2}} g_{\varepsilon}^{\mathrm{H}}, \mu_{L}\right)+\varepsilon^{\mathbf{m}+\mathbf{q}} Q^{H L}\left(\mu_{\mathrm{H}}^{\frac{1}{2}} g_{\varepsilon}^{\mathrm{H}}, \mu_{\mathrm{L}}^{\frac{1}{2}} g_{\varepsilon}^{\mathrm{L}}\right)\right\}
\end{aligned}
$$

For notational convenience, we set:

$$
\begin{array}{cc}
\mathcal{L}_{1}^{\mathrm{LH}}\left(g_{\epsilon}^{\mathrm{LH}}\right)=\left(\mu_{\mathrm{L}}\right)^{-1 / 2} Q^{L H}\left(\mu_{L}, \mu_{H}^{1 / 2} g_{\epsilon}^{H}\right), & \mathcal{L}_{2}^{\mathrm{LH}}\left(g_{\epsilon}^{\mathrm{LH}}\right)=\left(\mu_{\mathrm{L}}\right)^{-1 / 2} Q^{L H}\left(\mu_{L}^{1 / 2} g_{\epsilon}^{L}, \mu_{H}\right) \\
\mathcal{H}_{1}^{\mathrm{HL}}\left(g_{\epsilon}^{\mathrm{HL}}\right)=\left(\mu_{\mathrm{H}}\right)^{-1 / 2} Q^{H L}\left(\mu_{H}, \mu_{\mathrm{L}}^{\frac{1}{2}} g_{\varepsilon}^{\mathrm{L}}\right) & \mathcal{H}_{2}^{\mathrm{HL}}\left(g_{\epsilon}^{\mathrm{HL}}\right)=\left(\mu_{\mathrm{H}}\right)^{-1 / 2} Q^{L H}\left(\mu_{\mathrm{H}}^{\frac{1}{2}} g_{\varepsilon}^{\mathrm{H}}, \mu_{L}\right) \\
\Gamma_{1}^{\mathrm{LH}}\left(g_{\epsilon}^{\mathrm{LH}}\right)=\left(\mu_{\mathrm{H}}\right)^{-1 / 2} Q^{L H}\left(\mu_{\mathrm{L}}^{\frac{1}{2}} g_{\varepsilon}^{\mathrm{L}}, \mu_{\mathrm{H}}^{\frac{1}{2}} g_{\varepsilon}^{\mathrm{H}}\right), & \Gamma_{2}^{\mathrm{HL}}\left(g_{\epsilon}^{\mathrm{HL}}\right)=\left(\mu_{\mathrm{H}}\right)^{-1 / 2} Q^{H L}\left(\mu_{\mathrm{H}}^{\frac{1}{2}} g_{\varepsilon}^{\mathrm{H}}, \mu_{\mathrm{L}}^{\frac{1}{2}} g_{\varepsilon}^{\mathrm{L}}\right) .
\end{array}
$$

Here, $\varepsilon^{\gamma}$ allows us to choose the phenomenon we want to emphasize. By varying $\varepsilon, \beta$ we can formally derive the systems of the fluids dynamics.

The main result of the present paper is to perform the asymptotic limit when $\gamma=\beta=0$, $m=1$. Specifically the main result shows that we obtain compressible Euler equation for $F^{\mathrm{H}}$ and Incompressible Navier-Stokes equations for $F^{\mathrm{L}}$. The above formal derivation can be stated more precisely as follows. 
Theorem 3.1. Let $F_{\varepsilon}^{H}$ be smooth and assume that it converges to a limit, say $F_{0}^{H}$, in a sufficiently strong norm. Then, when $\gamma=\beta=0, m=1$, the limit of Eq. (3.9a) is a local Maxwellian

$$
F_{0}^{H}=\mathcal{M}\left[n^{H}, u, \theta\right]
$$

and the fluid quantities $\left(n^{H}, u, \theta\right)$ are governed by the compressible Euler equation

$$
\left\{\begin{array}{l}
\partial_{t} n^{H}+\operatorname{div}_{x}\left(n^{H} u\right)=0 \\
\partial_{t}\left(m^{H} n^{H} u\right)+\nabla_{x} \cdot\left(m^{H} n^{H} u \otimes u\right)+\nabla_{x} p^{H}=0 \\
\partial_{t} \mathcal{W}^{H}+\nabla_{x} \cdot\left(\left(\mathcal{W}^{H}+p^{H}\right) u\right)=0 .
\end{array}\right.
$$

Moreover, the fluid quantities $\left(n^{L}, u, \theta\right)$ satisfy the incompressible Navier-Stokes-Fourier equations

$$
\begin{gathered}
\left\{\begin{array}{l}
\operatorname{Div}_{x}\left(\left(n^{L}+\theta\right) I\right)=\nabla_{x}\left(n^{L}+\theta\right)=0 \\
\partial_{t} u+u \cdot \nabla_{x} u-\nu \Delta_{x} u=-\nabla_{x} p^{L} \\
\frac{5}{2}\left(\partial_{t} \theta+u \cdot \nabla \theta\right)-\kappa \Delta \theta=0
\end{array}\right. \\
\text { where } u=\left\langle g^{L} v\right\rangle \text { and } \theta=-n^{L}=\left\langle\left(\frac{|v|^{2}}{5}-1\right) g\right\rangle \text { and } \\
\kappa=\frac{1}{3} \int_{\mathbb{R}^{3}} B \cdot \mathbb{L}(B) \mu d v,
\end{gathered}
$$

where $\mu>0$ is the kinematic viscosity and $\kappa>0$ is the thermal diffusivity.

Corollary 3.2. Let $F_{\varepsilon}^{H}$ be smooth and assume that it converges to a limit, say $F_{0}^{H}$, in a sufficiently strong norm. Then, when $\gamma=\beta=0, m>1$, the fluid quantities $\left(n^{L}, u, \theta\right)$ satisfy the Stokes equation, that is:

$$
\left\{\begin{array}{l}
\partial_{t} u+\nabla_{x} p=\mu \Delta_{x} u \\
\operatorname{div}_{x} u=0 \\
\frac{5}{2} \partial_{t} u=\kappa \Delta_{x} \theta
\end{array}\right.
$$

This is a system of linear heat equations where the kinematic viscosity $\mu$ and the thermal diffusivity $\kappa$ have the same values as in the Navier-Stokes-Fourier system.

\section{Proof of Theorem 3.1}

This section is devoted to the proof of the main result of the present paper. As already mentioned in the introduction, the method for deriving the hydrodynamic equations from the Boltzmann equation differs from the Hilbert and Chapman-Enskog expansions.

Our proof of the Theorem 3.1 consists in the following five steps:

(1) Showing the asymptotic fluctuations;

(2) Establishing the incompressibility and Boussinesq's relations;

(3) Evaluating the limit for moments of the form $\left\langle\mathcal{L} \xi g_{\varepsilon}\right\rangle / \varepsilon$ for every $\xi \in \operatorname{Dom}(\mathcal{L}) \cap$ $\operatorname{Null}(\mathcal{L})^{\perp}$

(4) Determining the motion equation and heat equations;

(5) Determining Euler equation for the species $\mathrm{H}$ and Navier-Stokes and Stokes equations for species L.

Before starting the derivation of our differents equations, let's note that from $g_{\varepsilon} \rightarrow g$ in $w-L^{2}\left([0, T] ; L^{2}(\mathcal{M} d v)\right)$, it follows that the velocity moments $\varphi g_{\varepsilon}$ converge to $\langle\varphi g\rangle$ in $w-L^{2}\left([0, T] ; L^{2}(d x)\right)$ and hence in the sense of distributions, for any $\varphi$ polynomial in $v$.

Limit for the species $H$. 
Acting directly on the system of conservation laws deduced from Boltzlann mixture equation for the species $\mathrm{H}$, we will show first that the moments of $F_{0}^{\mathrm{H}}$, the weak limit of the sequence $F_{\varepsilon}^{\mathrm{H}}$, solve the compressible Euler system.

We divide this part of the proof into two main steps.

Step 1. Convergence to local equilibrium. Writing (3.9) as

$$
\varepsilon\left(\partial_{t} F_{\varepsilon}^{\mathrm{H}}+v \cdot \nabla_{x} F_{\varepsilon}^{\mathrm{H}}\right)=Q\left(F_{\varepsilon}^{\mathrm{H}}, F_{\varepsilon}^{\mathrm{H}}\right)+\varepsilon^{q+1} Q\left(F_{\varepsilon}^{\mathrm{H}}, F_{\varepsilon}^{\mathrm{L}}\right)
$$

and assuming that $F_{\varepsilon}^{\mathrm{H}}$ are sufficiently smooth and converge to a limit, say $F_{0}^{\mathrm{H}}$, in a sufficiently strong norm, we deduce that

$$
Q\left(F_{\varepsilon}^{\mathrm{H}}, F_{\varepsilon}^{\mathrm{H}}\right)=0 \quad \Longrightarrow \quad F^{\mathrm{H}}=\mathcal{M}\left[n^{\mathrm{H}}, u, \theta\right]
$$

for some $\left(n^{\mathrm{H}}, u, \theta\right)$ which can be function of $t$ and $x$.

Step 2. Passing to the limit in the local conservation laws. Taking the inner product $\langle\cdot\rangle$ of (4.1) with the collision invariants of $Q^{H H}$, one deduces:

$$
\begin{aligned}
& \partial_{t} n_{\varepsilon}^{\mathrm{H}}+\nabla_{x} \cdot\left(n_{\varepsilon}^{\mathrm{H}} u_{\varepsilon}\right)=0 \\
& \partial_{t}\left(m^{\mathrm{H}} n_{\varepsilon}^{\mathrm{H}} u_{\varepsilon}\right)+\nabla_{x}\left(m^{\mathrm{H}} n_{\varepsilon}^{\mathrm{H}} u_{\varepsilon} \otimes u_{\varepsilon}\right)+\nabla_{x} p_{\varepsilon}^{\mathrm{H}}=\varepsilon^{q} \mathcal{Q}^{\mathrm{HL}} \\
& \partial_{t} \mathcal{W}_{\varepsilon}^{\mathrm{H}}+\nabla_{x} \cdot\left(\mathcal{W}_{\varepsilon}^{\mathrm{H}} u_{\varepsilon}+\mathbb{P}_{\varepsilon}^{\mathrm{H}} u_{\varepsilon}+\mathbb{Q}_{\varepsilon}^{\mathrm{H}}\right)=\varepsilon^{q} \mathscr{Q}^{\mathrm{HL}} .
\end{aligned}
$$

Additionally, we obviously have that

$$
n_{\varepsilon}^{\mathrm{H}} \underset{\varepsilon \rightarrow 0}{\longrightarrow} n^{\mathrm{H}}, \quad u_{\varepsilon} \underset{\varepsilon \rightarrow 0}{\longrightarrow} u .
$$

We also see that

$$
\mathcal{W}_{\varepsilon}^{\mathrm{H}} \underset{\varepsilon \rightarrow 0}{\longrightarrow} \mathcal{W}^{\mathrm{H}}:=n^{\mathrm{H}}\left(m^{\mathrm{H}} \frac{|u|^{2}}{2}+\frac{3}{2} \kappa \theta\right) .
$$

Finally, a direct calculation gives

$$
\mathbb{P}_{\varepsilon}^{\mathrm{H}} \rightarrow \mathbb{P}^{\mathrm{H}}:=n^{\mathrm{H}} \kappa_{B} \theta \mathbf{I}=p^{\mathrm{H}} \mathbf{I} .
$$

Letting $\varepsilon \rightarrow 0$, in (4.3), one concludes that

$$
\begin{aligned}
& \partial_{t} n^{\mathrm{H}}+\operatorname{div}_{x}\left(n^{\mathrm{H}} u\right)=0, \\
& \partial_{t}\left(m^{\mathrm{H}} n^{\mathrm{H}} u\right)+\nabla_{x} \cdot\left(m^{\mathrm{H}} n^{\mathrm{H}} u \otimes u\right)+\nabla_{x} p^{\mathrm{H}}=0, \\
& \partial_{t} \mathcal{W}^{\mathrm{H}}+\nabla_{x} \cdot\left(\left(\mathcal{W}^{\mathrm{H}}+p^{\mathrm{H}}\right) u\right)=0 .
\end{aligned}
$$

Limit for the species $L$.

In terms of the relative number density fluctuation $f_{\varepsilon}^{\mathrm{L}}$, and after dividing by $\varepsilon$, we obtain

$$
\partial_{t} g_{\epsilon}^{L}+\frac{v}{\varepsilon} \cdot \nabla_{x} g_{\epsilon}^{L}=\frac{1}{\varepsilon^{2}} \mathbb{L}\left(g_{\epsilon}^{L}\right)+\varepsilon^{m-2} \Gamma\left(g_{\epsilon}^{L}\right)+\epsilon^{q-m} \mathcal{L}_{1}^{\mathrm{LH}}\left(g_{\epsilon}^{\mathrm{LH}}\right)+\epsilon^{q} \mathcal{L}_{2}^{\mathrm{LH}}\left(g_{\epsilon}^{\mathrm{HL}}\right)+\epsilon^{q} \Gamma_{1}^{\mathrm{LH}}\left(g_{\epsilon}^{\mathrm{LH}}\right) .
$$

The local conservation laws are written in terms of the relative number density fluctuation $g_{\varepsilon}^{\mathrm{L}}$ defined by $(3.15)$ as

$$
\begin{gathered}
\varepsilon \partial_{t}\left\langle g_{\varepsilon}^{\mathrm{L}}\right\rangle+\operatorname{div}_{x}\left\langle v g_{\varepsilon}\right\rangle=0 \\
\varepsilon \partial_{t}\left\langle v g_{\varepsilon}^{\mathrm{L}}\right\rangle+\operatorname{div}_{x}\left\langle v \otimes v g_{\varepsilon}\right\rangle=\mathcal{R}_{\varepsilon, 1}^{\mathrm{L}} \\
\varepsilon \partial_{t}\left\langle\frac{1}{2}|v|^{2} g_{\varepsilon}^{\mathrm{L}}\right\rangle+\operatorname{div}_{x}\left\langle v \frac{1}{2}|v|^{2} g_{\varepsilon}^{\mathrm{L}}\right\rangle=\mathcal{R}_{\varepsilon, 2}^{\mathrm{L}}
\end{gathered}
$$

where

$$
\left.\mathcal{R}_{\varepsilon, 1}^{\mathrm{L}}=\varepsilon^{\mathbf{q}+\mathbf{1}}\left\langle v \mathcal{L}_{1}^{\mathrm{LH}}\left(g_{\epsilon}^{\mathrm{LH}}\right)\right)\right\rangle+\varepsilon^{\mathbf{q}}\left\langle v \mathcal{L}_{2}^{\mathrm{LH}}\left(g_{\epsilon}^{\mathrm{LH}}\right)\right\rangle+\varepsilon^{\mathbf{q}}\left\langle v \Gamma_{1}^{\mathrm{LH}}\left(g_{\epsilon}^{\mathrm{LH}}\right)\right\rangle
$$

and

$$
\left.\mathcal{R}_{\varepsilon, 2}^{\mathrm{L}}=\varepsilon^{\mathbf{q}+\mathbf{1}}\left\langle\frac{1}{2}|v|^{2} \mathcal{L}_{1}^{\mathrm{LH}}\left(g_{\epsilon}^{\mathrm{LH}}\right)\right)\right\rangle+\varepsilon^{\mathbf{q}}\left\langle\frac{1}{2}|v|^{2} \mathcal{L}_{2}^{\mathrm{LH}}\left(g_{\epsilon}^{\mathrm{LH}}\right)\right\rangle+\varepsilon^{\mathbf{q}}\left\langle\frac{1}{2}|v|^{2} \Gamma_{1}^{\mathrm{LH}}\left(g_{\epsilon}^{\mathrm{LH}}\right)\right\rangle .
$$

The argument is split in several steps. 
Step 1: The limiting number density fluctuations. The first step shows that the limit $g$ is an infinitesimal Maxwellian.

Upon multiplying the equation (4.8) by $\varepsilon$ and letting $\varepsilon \rightarrow 0$, this suggests that

$$
g_{\varepsilon} \rightarrow g \text { in the sense of distributions on } \mathbb{R}^{*+} \times \mathbb{R}^{3} \times \mathbb{R}^{3} \text { with } \quad \mathbb{L} g=0
$$

which says that

$$
\operatorname{Ker}(\mathbb{L})=\left\{g^{\mathrm{L}}, g^{\mathrm{L}}=a+b \cdot v+c|v|^{2}, \quad \text { where } \quad(a, b, c) \in \mathbb{R} \times \mathbb{R}^{3} \times \mathbb{R}\right\} .
$$

By Hilbert's theorem, $g^{\mathrm{L}}$ is an infinitesimal Maxwellian, meaning that $g^{\mathrm{L}}(t, x, v)$ is of the form

$$
g^{\mathrm{L}}=\left\{n^{\mathrm{L}}+u \cdot v+\theta\left(\frac{|v|^{2}}{2}-\frac{3}{2}\right)\right\} \sqrt{\mu_{\mathrm{L}}}
$$

for some $\left(n^{\mathrm{L}}, u, \theta\right)$ in $L^{\infty}\left(d t ; L^{2}\left(d x ; \mathbb{R} \times \mathbb{R}^{3} \times \mathbb{R}\right)\right)$.

Step 2: The incompressibility condition. The second step shows that $\left(n^{\mathrm{L}}, u, \theta\right)$ satisfies the incompressibility and Boussinesq relations.

Passing to the limit in the sense of distributions in the continuity equation, i.e. the first equality in (4.9), we obtain

$$
\operatorname{div}_{x}\langle v g\rangle=0 \quad \text { or equivalently } \quad \operatorname{div}_{x} u=0
$$

which is the incompressibility condition in the Navier-Stokes equations.

Passing to the limit in the sense of distributions in the momentum equation, i.e. the second equality in (4.9), we obtain

$$
\operatorname{div}_{x}\langle v \otimes v g\rangle=0 \quad \text { or equivalently } \quad \operatorname{div}_{x}\left(n^{\mathrm{L}}+u\right)=0 .
$$

Since $g \in L^{\infty}\left(d t ; L^{2}(\mu d v d x)\right), n^{\mathrm{L}}+\theta \in L^{\infty}\left(d t ; L^{2}(x)\right)$; the only a.e. constant function in $L^{2}\left(\mathbb{R}^{3}\right)$ being 0 . The Boussinesq relation implies that the infinitesimal Maxwellian form (4.12) of $g$ reduces to

$$
n^{\mathrm{L}}+\theta=0 \quad \text { and } \quad g^{\mathrm{L}}=v \cdot u+\left(\frac{|v|^{2}}{2}-\frac{5}{2}\right) \theta,
$$

for some $(u, \theta) \in L^{\infty}\left(d t ; L^{2}\left(d x ; \mathbb{R}^{3} \times \mathbb{R}\right)\right)$.

Step 3: The limiting fluctuations of density and temperature. The next three steps show that the evolution of $(u, \theta)$ is governed by the motion and heat equations. The difficulty here is that when the local conservation laws (4.9) are written so that the time derivatives are order 1 , the fluxes become order $1 / \epsilon$.

Note that the asymptotic limit can be formally derived from (3.16) using the asymptotic expansion

$$
g_{\epsilon}^{\mathrm{L}}=g^{\mathrm{L}}+\varepsilon h+\varepsilon^{2} k+O\left(\varepsilon^{3}\right) .
$$

Besides, to first order, we obtain

$$
\partial_{t} g^{\mathrm{L}}+v \cdot \nabla_{x} h=-\mathbb{L} k+2 \Gamma^{\mathrm{L}}\left(g^{\mathrm{L}}, h\right)
$$

from which we deduce that

$$
\begin{aligned}
& \partial_{t}\left\langle v g^{\mathrm{L}}\right\rangle+\nabla_{x} \cdot\langle v \otimes v h\rangle=0, \\
& \partial_{t}\left\langle\left(\frac{|v|^{2}}{5}-1\right) g^{\mathrm{L}}\right\rangle+\nabla_{x} \cdot\left\langle v\left(\frac{|v|^{2}}{5}-1\right) h\right\rangle=0 .
\end{aligned}
$$

To get a closed equation for $g^{\mathrm{L}}$, we have to inverse the operator $\mathbb{L}$.

The next Lemma claims that the integral equation $\mathbb{L} \phi=\psi, \psi \in L^{2}(\mu d v)$ satisfies the Fredholm alternative. 
Lemma 4.1. Let $A=v \otimes v-\frac{1}{3}|v|^{2} \mathbf{I}$ (resp. $\left.B=\left(\frac{|v|^{2}}{2}-\frac{5}{2}\right) v\right)$ in $L^{2}\left((\mu d v) \cap \operatorname{Null}^{\perp}(\mathbb{L})\right)$. The Fredholm alternative holds for $\mathbb{L}$ namely, that Range $(\mathbb{L})=\operatorname{Null}(\mathbb{L})^{\perp}$. In particular, it implies that unique $\hat{A} \in L^{2}\left(\mu d v ; \mathbb{R}^{3 \times 3}\right)$ and $\hat{B} \in L^{2}\left(\mu d v ; \mathbb{R}^{3}\right)$ exist which solve

$$
\begin{array}{ll}
\mathbb{L} \hat{A}=A & \hat{A} \in \operatorname{Null}^{\perp}(\mathbb{L}) \text { entrywise } \\
\mathbb{L} \hat{B}=B & \hat{B} \in \operatorname{Null}^{\perp}(\mathbb{L}) \text { entrywise. }
\end{array}
$$

The solution $\hat{A}$ or $\hat{B}$ of the equation (4.19a) can then be written as

$$
\hat{A}(v)=\gamma(|v|) A(v) \text {. }
$$

The higher order terms of $\varepsilon$ tend to zero, when $\varepsilon \rightarrow 0$. We finally obtain in the limit:

$$
\begin{aligned}
& \partial_{t}\left\langle g^{\mathrm{L}} v_{i}\right\rangle+\nabla_{x} \cdot\left\langle A_{i j}\left(\Gamma\left(g^{\mathrm{L}}, g^{\mathrm{L}}\right)-v \cdot \nabla g^{\mathrm{L}}\right)\right\rangle+\nabla\left\langle\frac{|v|^{2}}{3} h\right\rangle . \\
& \partial_{t}\left\langle\left(\frac{|v|^{2}}{5}-1\right) g^{\mathrm{L}}\right\rangle+\nabla_{x} \cdot\left\langle\hat{B}\left(\Gamma\left(g^{\mathrm{L}}, g^{\mathrm{L}}\right)-v \cdot \nabla g^{\mathrm{L}}\right)\right\rangle=0 .
\end{aligned}
$$

The crucial ingredient needed to compute the term $\left\langle A_{i j}\left(\Gamma\left(g^{\mathrm{L}}, g^{\mathrm{L}}\right)\right)\right\rangle$ in Eq. (4.21a) is the following result whose proof can be found in [4].

Lemma 4.2. For each $\phi, \psi \in \mathbb{L}_{\mathcal{M}}$, one has

$$
\Gamma_{\mathcal{M}}(\phi, \psi)=\frac{1}{2} \mathbb{L}_{\mathcal{M}}(\phi \psi) .
$$

Proof. Differentiate twice the relation $C\left(\mathcal{M}_{(n, u, \theta)}\right)=0$, and observe that the range of the differential $d \mathcal{M}_{(n, u, \theta)}$ is equal to $\operatorname{Ker}(\mathbb{L})$.

Bearing all above in mind, one has

$$
\left\langle\hat{A} \Gamma\left(g^{\mathrm{L}}, g^{\mathrm{L}}\right\rangle=\frac{1}{2}\left\langle\hat{A} \mathbb{L}\left(\left(g^{\mathrm{L}}\right)^{2}\right)\right\rangle=\frac{1}{2}\left\langle\hat{A}\left(g^{\mathrm{L}}\right)^{2}\right\rangle=\frac{1}{2}\langle\hat{A} \otimes \hat{A}\rangle:\left(u \otimes u-\frac{1}{3}|u|^{2} I\right) .\right.
$$

Therefore

$$
\left\langle\hat{A} \Gamma\left(g^{\mathrm{L}}, g^{\mathrm{L}}\right\rangle \rightarrow \frac{1}{2}\langle A \otimes A\rangle:\left(u \otimes u-\frac{1}{3}|u|^{2} I\right)-\frac{1}{2}\langle A \otimes A\rangle: D(u) .\right.
$$

Thus, in view of the previous computation, the second term in (4.21a) becomes

$$
\begin{aligned}
\nabla_{x} \cdot\left\langle A_{i j}\left(\Gamma\left(g^{\mathrm{L}}, g^{\mathrm{L}}\right)-v \cdot \nabla g^{\mathrm{L}}\right)\right\rangle & \rightarrow \frac{1}{2}\langle A \otimes A\rangle:\left(u \otimes u-\frac{1}{3}|u|^{2} I\right)-\frac{1}{2}\langle\hat{A} \otimes A\rangle: D(u) \\
& =\left(u \otimes u-\frac{1}{3}|u|^{2} I\right)-\nu D(u)
\end{aligned}
$$

where $\nu$ is defined by

$$
\nu=\frac{1}{10}\langle\hat{A}: \mathbb{L} \hat{A}\rangle .
$$

Upon placing this expression in the momentum conservation laws, we obtain

$$
\partial_{t} u+\operatorname{div}_{x}(u \otimes u)-\nu \operatorname{div}_{x} D(u)+\operatorname{div}_{x}\left(\frac{1}{3}|u|^{2} I\right)+\nabla_{x} \frac{1}{\varepsilon}\left\langle\frac{1}{3}|v|^{2} g_{\varepsilon}\right\rangle=0,
$$

or equivalently

$$
\partial_{t} u+\operatorname{div}_{x}(u \otimes u)-\nu \Delta_{x} u=-\nabla_{x}\left(\frac{1}{\varepsilon}\left\langle\frac{1}{3}|v|^{2} g_{\varepsilon}\right\rangle-\frac{1}{3}|u|^{2}\right) .
$$

Indeed, the divergence free condition $\operatorname{div}_{x} u=0$ implies that

$$
\operatorname{div}_{x} D(u)=\Delta_{x} u+\nabla_{x}\left(\operatorname{Div}_{x} u\right)-\frac{2}{3} \nabla_{x}\left(\operatorname{div}_{x} u\right)=\Delta_{x} u .
$$

Equivalently

$$
\partial_{t} u+\operatorname{div}_{x}(u \otimes u)-\nu \Delta_{x} u=0 \text { (modulo gradient fields) }
$$

which is Navier-Stokes equation and the Fourier equation namely (3.23).

Case $m>1$. Derivation of the Stokes equation (the momentum equation) for L.

Step 1. The starting point in our analysis is Eq. (4.8). 
Accordingly, we split the tensor $v \otimes v$ into its traceless and scalar component:

$$
v \otimes v=\left(v \otimes v-\frac{1}{3}|v|^{2} I\right)+\frac{1}{3}|v|^{2} I,
$$

so that the local conservation of momentum becomes

$$
\begin{aligned}
\partial_{t}\left\langle v g_{\epsilon}^{L}\right\rangle+\frac{1}{\varepsilon} \nabla_{x} & \left.\cdot\left\langle\left(v \otimes v-\frac{1}{3} \mathbf{I}\right) g_{\epsilon}\right)\right\rangle+\frac{1}{\varepsilon} \nabla_{x}\left\langle\frac{1}{3}|v|^{2} g_{\varepsilon}\right\rangle \\
& =\epsilon^{q-m}\left\langle\mathcal{L}_{1}^{\mathrm{LH}}\left(g_{\epsilon}^{\mathrm{LH}}\right)\right\rangle+\epsilon^{q}\left\langle\mathcal{L}_{2}^{\mathrm{LH}}\left(g_{\epsilon}^{\mathrm{HL}}\right)\right\rangle+\epsilon^{q}\left\langle\Gamma_{1}^{\mathrm{LH}}\left(g_{\epsilon}^{\mathrm{LH}}\right)\right\rangle
\end{aligned}
$$

where the trace of the tensor $v \otimes v$ has been separated out. The term $\nabla_{x}\left\langle\frac{1}{3}|v|^{2} g_{\varepsilon}\right\rangle$ has the form $\nabla_{x} p_{\varepsilon}$ for $p_{\varepsilon} \equiv \frac{1}{3}|v|^{2} g_{\varepsilon}$ it vanishes when, according to our prescription, we integrate (4.22) against a divergence-free test function. Therefore, we are going to ignore this term in the computation below.

The goal is to replace the second term in the equation (4.22) with an expression whose limit we can compute. To this end let's now define

$$
\Phi(v)=\mathbb{L}^{-1}\left(v \otimes v-\frac{1}{3}|v|^{2} \mathbf{I}\right) .
$$

Next, multiply (4.22) by $\Phi$ and average it over the velocity domain. Using the selfadjointness of $\mathbb{L}$, we obtain

$$
\begin{aligned}
\varepsilon \partial_{t}\left\langle\Phi(v) g_{\varepsilon}\right\rangle+\nabla_{x} \cdot\left\langle v \otimes \Phi g_{\varepsilon}\right\rangle=- & \frac{1}{\varepsilon}\left\langle\left(v \otimes v-\frac{1}{3}|v|^{2} \mathbf{I}\right) g_{\varepsilon}\right\rangle+\epsilon^{q-m}\left\langle\mathcal{L}_{1}^{\mathrm{LH}}\left(g_{\epsilon}^{\mathrm{LH}}\right) \Phi\right\rangle \\
& +\epsilon^{q}\left\langle\mathcal{L}_{2}^{\mathrm{LH}}\left(g_{\epsilon}^{\mathrm{HL}}\right) \Phi\right\rangle+\epsilon^{q}\left\langle\Gamma_{1}^{\mathrm{LH}}\left(g_{\epsilon}^{\mathrm{LH}}\right) \Phi\right\rangle .
\end{aligned}
$$

Upon formally letting $\varepsilon \rightarrow 0$ in this equation, only the second and third terms do not vanish, so that

$$
\nabla_{x} \cdot\left\langle v \otimes \Phi g_{\varepsilon}\right\rangle=-\frac{1}{\varepsilon}\left\langle\left(v \otimes v-\frac{1}{3}|v|^{2} \mathbf{I}\right) g_{\varepsilon}\right\rangle+O(\varepsilon) .
$$

One can then pass to the limit in (4.8) to get

$$
\partial_{t}\langle v g\rangle-\nabla_{x} \cdot\left(\nabla_{x} \cdot\langle v \otimes \Phi g\rangle\right)=0 .
$$

To conclude, we need to compute the term $\nabla_{x} \cdot\langle v \otimes \Phi g\rangle$. Observe that

$$
\nabla_{x} \cdot\langle v \otimes \Phi g\rangle=\left\langle\mathbb{L}^{-1}\left(v \otimes v-\frac{1}{3}|v|^{2} \mathbf{I}\right) \otimes(v \otimes v): \nabla_{x} u .\right.
$$

One can add to the expression (4.26), without altering it, the term $-\frac{1}{3}|v|^{2} \mathbf{I}$ since the latter is in $\operatorname{Null}(\mathbb{L})$, which is orthogonal to the range of $\mathbb{L}^{-1}$. We can rewrite

$$
\nabla_{x} \cdot\langle v \otimes \Phi g\rangle=\left\langle\mathbb{L}^{-1}\left(v \otimes v-\frac{1}{3}|v|^{2} \mathbf{I}\right) \otimes\left(v \otimes v-\frac{1}{3}|v|^{2} \mathbf{I}\right): \nabla_{x} u .\right.
$$

By virtue of (4.20), we can set

$$
\begin{aligned}
\left\langle\mathbb{L}^{-1}(v \otimes v-\right. & \left.\left.\frac{1}{3}|v|^{2} \mathbf{I}\right) \otimes\left(v \otimes v-\frac{1}{3}|v|^{2} \mathbf{I}\right)\right\rangle \\
& =\left\langle\beta(|v|)\left(v \otimes v-\frac{1}{3}|v|^{2} \mathbf{I}\right) \otimes\left(v \otimes v-\frac{1}{3}|v|^{2} \mathbf{I}\right)\right\rangle \equiv \mathcal{T}
\end{aligned}
$$

where $\mathcal{T}$ is fourth order tensor. Taking into account the fact that $\mathbb{L}^{-1}$ is a positive definite operator, the diagonal entries of $\mathcal{T}$ are positive quantities, as one expects them to be, given their physical interpretation in relation with the kinematic viscosity $\nu$, as we are going to see. Componentwise, we have

$$
\mathcal{T}_{i j l m}=\left\langle\beta(|v|)\left(v_{i} v_{j}-\frac{1}{3}|v|^{2} \delta_{i j}\right)\left(v_{l} v_{m}-\frac{1}{3}|v|^{2} \delta_{l m}\right)\right\rangle
$$

which, because of the oddness of the integrand, vanishes whenever there is an unpaired index. The many symmetries of the tensor $\mathcal{T}$ allow to write, by inspection,

$$
\mathcal{T}_{i j l m}=a \delta_{i j} \delta_{l m}+b \delta_{i l} \delta_{j m}+c \delta_{i m} \delta_{j l} .
$$


But $\mathcal{T}_{i j l m}=\mathcal{T}_{i j m l}$ implies $c=b$, so $\mathcal{T}_{i j l m}=c\left(\delta_{i m} \delta_{j l}+\delta_{i l} \delta_{j m}\right)+a \delta_{i j} \delta_{l m}$. Since $\left(v \otimes v-\frac{1}{3}|v|^{2} \mathbf{I}\right)$ is a traceless tensor, it has to be that $\mathcal{T}_{i j l l}=l l i j=0$, which yields $2 c \delta_{i l} \delta_{j l}+a \delta_{i j} \delta_{l l}=0$, that is $a=-\frac{1}{3} c$, so that

$$
\mathcal{T}_{i j l m}=c\left(\delta_{i m} \delta_{j l}+\delta_{i l} \delta_{j m}-\frac{2}{3} \delta_{i j} \delta_{l m}\right) .
$$

The value of $c$ is obtained by taking the double trace of $\mathcal{T}$. One writes $\mathcal{T}_{i j i j}=20 c$, from which the positivity of $c$ follows.

To compute $\left(\mathcal{T}: \nabla_{x} u\right)$, notice that $\delta_{i m} \delta_{j l} \partial_{l} u_{m}=\partial_{j} u_{i}, \delta_{i l} \delta_{j m} \partial_{l} u_{m}=\partial_{i} u_{j}$ while the incompressibility condition yields $\delta_{i j} \delta_{l m} \partial_{l} u_{m}=0$. Therefore one gets

$$
\mathcal{T}: \nabla_{x} u=c\left(\nabla_{x} u+\left(\nabla_{x} u\right)^{T}\right) .
$$

Taking the divergence of the expression (4.27), we finally obtain

$$
\nabla_{x} \cdot\left(\nabla_{x} \cdot\langle v \otimes \Phi g\rangle\right)=c \nabla_{x} \cdot\left(\nabla_{x} u+\left(\nabla_{x} u\right)^{T}\right)=\nu \Delta u(t, x),
$$

where we used the incompressibilty condition and set $c=\nu$.

From equation (4.25) because of the weak notion of solution derived from the Boltzmann equation, one has

$$
\frac{d}{d t} \int \phi \cdot u d x=\nu \int \Delta_{x} \phi \cdot u d x
$$

valid for any $\phi \in \mathcal{V}=\left\{u \in\left(C^{\infty}(\Omega)\right)^{3}, \nabla_{x} \cdot u=0, \int u d x=0\right\}$, where $\Omega \subset \mathbb{R}^{3}$, so that, for almost every $t_{1}, t_{2}$ the relation

$$
\int \phi \cdot u\left(t_{2}\right) d x-\int \phi \cdot u\left(t_{1}\right) d x-\nu \int_{t_{1}}^{t_{2}} \int \Delta_{x} \phi \cdot u d t d x=0
$$

holds. One can therefore write

$$
\partial_{t} u(t, x)+\nabla_{x} p(t, x)=\nu \Delta_{x} u(t, x) .
$$

To obtain the heat equation we start with equation (4.12). One easily checks that

$$
\left.\left.\left\langle\frac{1}{2}|v|^{2}-5\right) g_{\varepsilon}^{\mathrm{L}}\right\rangle \rightarrow\left\langle\frac{1}{2}|v|^{2}-5\right) g^{\mathrm{L}}\right\rangle=\frac{5}{2} \theta
$$

and one finds that

$$
\left\langle\hat{B} v \cdot \nabla_{x} g^{\mathrm{L}}\right\rangle=\left\langle\frac{5}{2} \kappa \nabla_{x} \theta\right\rangle
$$

and like

$$
\left\langle\hat{B} Q\left(g^{\mathrm{L}}, g^{\mathrm{L}}\right)\right\rangle=\frac{1}{2}\left\langle\hat{B} \mathbb{L}_{\mu}\left(\left(g^{\mathrm{L}}\right)^{2}\right)\right\rangle=\frac{1}{2}\left\langle\left(\mathbb{L}_{\mu} \hat{B}\right)\left(g^{\mathrm{L}}\right)^{2}\right\rangle=\frac{1}{2}\left\langle B\left(g^{\mathrm{L}}\right)^{2}\right\rangle=\frac{5}{2} u \theta .
$$

Combining (4.29), (4.30) with (4.31), we thereby finish the proof of the first part of our theorem:

$$
\frac{5}{2} \partial_{t} \theta+\operatorname{div}(u \theta)-\kappa \Delta_{x} \theta=0
$$

with

$$
\kappa=\frac{2}{15}\langle\hat{B} \cdot \mathbb{L} \hat{B}\rangle
$$

Step 2. Derivation of the energy equation

This step shows that the evolution of $(u, \theta)$ is governed by the motion and heat equations (3.24). 
Combining the conservation laws for mass and energy

$$
\begin{aligned}
& \frac{5}{2}\left(\partial_{t}\left\langle g_{\varepsilon}\right\rangle+\frac{1}{\varepsilon} \nabla_{x} \cdot\left\langle v g_{\varepsilon}\right\rangle\right)=0 \\
& \partial_{t}\left\langle\frac{1}{2}|v|^{2} g_{\varepsilon}\right\rangle+\frac{1}{\varepsilon} \nabla_{x} \cdot\left\langle v g_{\varepsilon}\right\rangle=\mathcal{R}_{\varepsilon, 2}^{\mathrm{L}}
\end{aligned}
$$

yields

$$
\partial_{t}\left\langle\left(\frac{1}{2}|v|^{2}-\frac{5}{2}\right) g_{\varepsilon}\right\rangle+\frac{1}{\varepsilon} \nabla_{x} \cdot\left\langle\left(v \frac{1}{2}|v|^{2}-\frac{5}{2}\right) g_{\varepsilon}\right\rangle=\mathcal{R}_{\varepsilon, 2}^{\mathrm{L}} .
$$

Upon formally letting $\varepsilon \rightarrow 0$ in the equation (4.33), the first term of (4.33) converges to

$$
\begin{aligned}
\partial_{t} & \left\langle\left(\frac{1}{2}|v|^{2}-\frac{5}{2}\right)\left(n(t, x)+v \cdot u(t, x)+\left(\frac{1}{2}|v|^{2}-\frac{3}{2}\right) \theta(t, x)\right)\right\rangle \\
& \left.=\frac{3}{2} \partial_{t} \theta-\partial_{t}\left\langle n(t, x)+v \cdot u(t, x)+\left(\frac{1}{2}|v|^{2}-\frac{3}{2}\right) \theta(t, x)\right)\right\rangle \\
& =\frac{3}{2} \partial_{t} \theta-\partial_{t} n=\frac{5}{2} \partial_{t} \theta
\end{aligned}
$$

where we used $\left\langle\left(\frac{1}{2}|v|^{2}-\frac{3}{2}\right)^{2}\right\rangle=\frac{3}{2},\left\langle\left(\frac{1}{2}|v|^{2}-\frac{3}{2}\right)\right\rangle=0$ and the integrated Boussinesq relation $n=-\theta$.

For the second term of (4.33) we make use of a technique similar to the one employed for the corresponding term in the momentum equation, namely write

$$
\frac{1}{\varepsilon} \nabla_{x} \cdot\left\langle\left(v \frac{1}{2}|v|^{2}-\frac{5}{2}\right) g_{\varepsilon}\right\rangle=\frac{1}{\varepsilon}\left\langle\Psi \mathbb{L} g_{\varepsilon}\right\rangle,
$$

where now

$$
\Psi \equiv \mathbb{L}^{-1}\left[\left(\frac{1}{2}|v|^{2}-\frac{5}{2}\right) v\right]
$$

It can be checked that $\left(\frac{1}{2}|v|^{2}-\frac{5}{2}\right) v$ is in $\operatorname{Null}(\mathbb{L})^{\perp}$ and so it is in the range of $\mathbb{L}^{-1}$. Eliminate $\left\langle\Psi \mathbb{L} g_{\varepsilon}\right\rangle$ using the Boltzmann equation, which gives

$$
\left\langle\Psi \mathbb{L} g_{\varepsilon}\right\rangle=-\varepsilon\left\langle\Psi \partial_{t} g_{\varepsilon}\right\rangle-\left\langle\Psi v \cdot \nabla_{x} g_{\varepsilon}\right\rangle .
$$

The first term on the right-hand side goes to zero, while taking the limit in the second term and bringing the gradient outside, one sees that $\left\langle\Psi v \cdot \nabla_{x} g_{\varepsilon}\right\rangle$ converges to $\nabla_{x} \cdot\langle v \otimes \Psi g\rangle$. Direct calculation shows that

$$
\begin{aligned}
\nabla_{x} \cdot\langle v \otimes \Psi g\rangle & =\nabla_{x} \cdot\left\langle v \otimes \Psi\left(n(t, x)+v \cdot u(t, x)+\left(\frac{1}{2}|v|^{2}-\frac{5}{2}\right) \theta(t, x)\right)\right\rangle \\
& =\nabla_{x} \cdot\left\langle v \otimes \mathbb{L}^{-1}\left[\left(\frac{1}{2}|v|^{2}-\frac{5}{2}\right) v\right]\left(v \cdot u(t, x)+\left(\frac{1}{2}|v|^{2}-\frac{5}{2}\right) \theta(t, x)\right\rangle,\right.
\end{aligned}
$$

where again, the Boussinesq relation has been used. Since $\mathbb{L}^{-1}$ preserves the parity, the only surviving term is

$$
\begin{aligned}
& \nabla_{x} \cdot\left\langle v \otimes \mathbb{L}^{-1}\left[\left(\frac{1}{2}|v|^{2}-\frac{5}{2}\right) v\right]\left(\frac{1}{2}|v|^{2}-\frac{5}{2}\right) \theta(t, x)\right\rangle \\
= & \left\langle\mathbb{L}^{-1}\left[\left(\frac{1}{2}|v|^{2}-\frac{5}{2}\right) v\right] \otimes v\left(\frac{1}{2}|v|^{2}-\frac{5}{2}\right)\right\rangle \cdot \nabla_{x} \theta(t, x)=\frac{5}{2} \kappa \nabla_{x} \theta .
\end{aligned}
$$

It follows directly from Lemma 4.1 and relation (4.20) and the symmetry properties of the integrand, that

$$
\begin{aligned}
& \left\langle\mathbb{L}^{-1}\left[\left(\frac{1}{2}|v|^{2}-\frac{5}{2}\right) v\right] \otimes v\left(\frac{1}{2}|v|^{2}-\frac{5}{2}\right)\right\rangle \\
= & \left\langle\alpha(|v|)\left(\frac{1}{2}|v|^{2}-\frac{5}{2}\right) v \otimes v\left(\frac{1}{2}|v|^{2}-\frac{5}{2}\right)\right\rangle=\frac{5}{2} \kappa \mathbf{I},
\end{aligned}
$$


with

$$
\kappa=\frac{2}{15}\langle\Psi \cdot \mathbb{L} \Psi\rangle
$$

Taking the divergence of the expression (4.38) and gathering the limiting terms in (4.33), we finally get

$$
\partial_{t} \theta(t, x)=\kappa \Delta(t, x) .
$$

We have thus recovered the Stokes equation. This concludes the proof of the Theorem 3.1 and of the related corollary.

\section{Conclusions and Research Perspectives}

This paper has been concerned with a hydrodynamic limit for a binary inert gas mixture by employing a new mathematical method. This study reveals significant physical and mathematical features which result from the interaction of the component gases as described by the kinetic models. Our analysis has been limited to the case $\gamma=\beta=0, m \geq 1$ that has shown an important macroscopic dynamics related to the Navier-Stokes equations. The derivation of macroscopic equation for further general cases is the main research perspective.

In particular we are interested in showing that at the hydrodynamic scale the system shows that the flow of the mixture vanishes in the continuum limit, but the vanishing flow gives a finite effect on the behavior of the mixture in this limit. This is a kind of phenomena called "ghost effects" (the effect of an infinitesimal flow field on other physical quantities, such as the temperature field) discovered recently by Sone [33], [30] in the continuum limit that has been studied in [35].

The analysis of the ghost effect in a single component gas is one of the classical problems in modern kinetic theory and has been tackled by various methods, including moment methods. It is well known that rarefied gases, in which the molecular mean free path is not negligible compared to the typical scale of the flow, should be dealt with the Boltzmann equation and not by the system of conventional fluid-dynamic equations. The analysis of the latter equation is, however, not an easy task, mainly because of the complexity of the collision term, which represents effects of molecular interactions on a change of the velocity distribution function of molecules [31]. This effect is particularly important because it reveals the fatal defect contained in the Navier-Stokes system for a gas. The effect manifests itself in a wide class of problems.

Moreover we are interested in showing that the sequence: Euler equations, Navier-Stokes equations, Burnett equations, etc. breaks down at the Burnett level since these equations are ill-posed [12]. In fact, it is well known (see [12], [13], [14]) that the Burnett hydrodynamic equations violate the basic physics behind the Boltzmann equation. Namely, sufficiently

short acoustic waves are amplified with time instead of decaying.

The rigorous proofs are in progress and the results will be presented in due course.

Acknowledgement. CB was partially supported by L'Agence Nationale de la Recherche (ANR T-KiNeT Project).

\section{REFERENCES}

[1] P. Andries, K. Aoki, and B. Perthame. A consistent BGK-type model for gas mixtures. J. Statist. Phys., 106(5-6):993-1018, 2002.

[2] K. Aoki, C. Bardos, and S. Takata. Knudsen layer for gas mixtures. J. Statist. Phys., 112(3-4):629-655, 2003.

[3] C. Bardos, F. Golse, and C. Levermore. Sur les limites asymptotiques de la théorie cinétique conduisant à la dynamique des fluides incompressibles. C. R. Acad. Sci. Paris Sér. I Math., 309(11):727-732, 1989. 
[4] C. Bardos, F. Golse, and C. Levermore. Fluid dynamic limits of kinetic equations. I. Formal derivations. J. Statist. Phys., 63(1-2):323-344, 1991.

[5] C. Bardos, F. Golse, and C. Levermore. Fluid dynamic limits of kinetic equations. II. Convergence proofs for the Boltzmann equation. Comm. Pure Appl. Math., 46(5):667-753, 1993.

[6] S. Bastea, R. Esposito, J. L. Lebowitz, and R. Marra. Binary fluids with long range segregating interaction. I. Derivation of kinetic and hydrodynamic equations. J. Statist. Phys., 101(5-6):1087-1136, 2000.

[7] C. Bianca and C. Dogbe. Kinetic models coupled with Gaussian thermostats: macroscopic frameworks. Nonlinearity, 27(12):2771-2803, 2014.

[8] C. Bianca and A. Lemarchand. Density evolution by the low-field limit of kinetic frameworks with thermostat and mutations. Commun. Nonlinear Sci. Numer. Simul., 20(1):14-23, 2015.

[9] M. Bisi. Incompressible Navier-Stokes equations from Boltzmann equations for reacting mixtures. $J$. Phys. A, 47(45):455203, 16, 2014.

[10] M. Bisi and L. Desvillettes. Formal passage from kinetic theory to incompressible Navier-Stokes equations for a mixture of gases. ESAIM Math. Model. Numer. Anal., 48(4):1171-1197, 2014.

[11] M. Bisi, G. Martalò, and G. Spiga. Multi-temperature hydrodynamic limit from kinetic theory in a mixture of rarefied gases. Acta Appl. Math., 122:37-51, 2012.

[12] A. V. Bobylev. On the Chapman-Enskog and Grad methods for solving the Boltzmann equation. Dokl. Akad. Nauk SSSR, 262(1):71-75, 1982

[13] A. V. Bobylev. Generalized Burnett hydrodynamics. J. Stat. Phys., 132(3):569-580, 2008.

[14] A.V. Bobylev and A. Windfäll. Boltzmann equation and hydrodynamics at the Burnett level. Kinet. Relat. Models, 5(2):237-260, 2012.

[15] S. Chapman and T. G. Cowling. The Mathematical Theory of Non-uniform Gases. Cambridge University Press, Cambridge, 1939.

[16] P. Degond. Asymptotic continuum models for plasmas and disparate mass gaseous binary mixtures. In Gianfranco Capriz Paolo Maria Mariano, editor, Material Substructures in Complex Bodies, pages 1 62. Elsevier Science Ltd, Oxford, 2007.

[17] C. Dogbe. Fluid dynamic limits for gas mixture. I. Formal derivations. Math. Models Methods Appl. Sci., 18(9):1633-1672, 2008

[18] R. Esposito and M. Pulvirenti. From particles to fluids. In Handbook of mathematical fluid dynamics. Vol. III, pages 1-82. North-Holland, Amsterdam, 2004.

[19] J. Ferziger and H. G. Kaper. Mathematical theory of transport processes in gases. North-Holland Pub. Co., Amsterdam, 1972. Distributed in the U.S. by American Elsevier Pub. Co., New York.

[20] A. Fick. On liquid diffusion. Journal of Membrane Science, 100(1):33 - 38, 1995. The early history of membrane science selected papers celebrating vol. 100.

[21] E. Goldman and L. Sirovich. Equations for Gas Mixtures. Physics of Fluids (1958-1988), 10(9):19281940, 1967.

[22] H. Grad. On the kinetic theory of rarefied gases. Comm. Pure Appl. Math., 2:331-407, 1949.

[23] Y. Guo. The Vlasov-Maxwell-Boltzmann system near Maxwellians. Invent. Math., 153(3):593-630, 2003.

[24] J. O. Hirschfelder, C. F. Curtis, and R. B. Bird. Molecular Theory of Gases and Liquids. Wiley, New York, 1954.

[25] J. Jang. Vlasov-Maxwell-Boltzmann diffusive limit. Arch. Ration. Mech. Anal., 194(2):531-584, 2009.

[26] E. A. Johnson. Energy and momentum equations for disparate-mass binary gases. Physics of Fluids (1958-1988), 16(1):45-49, 1973.

[27] M. N. Kogan. Dynamik des verdünnten Gases. Kinetische Theorie. Moskau: Verlag 'Nauka', Hauptredaktion für physikalisch-mathematische Literatur. 440, 1967.

[28] S. Kosuge, K. Aoki, and S. Takata. Heat transfer in a gas mixture between two parallel plates: finitedifference analysis of the Boltzmann equation, volume 585 of Aip Conference Proceedings, pages 289296. Amer Inst Physics, Melville, 2001.

[29] L. Sirovich and J. K. Thurber. On some mathematical aspects of kinetic model equations. Quart. Appl. Math., 25:175-186, 1967.

[30] Y. Sone. Continuum gas dynamics in the light of kinetic theory and new features of rarefied gas flows. Adv. Mech., 27(3):389-404, 1997.

[31] Y. Sone. Molecular gas dynamics. Modeling and Simulation in Science, Engineering and Technology. Birkhäuser Boston, Inc., Boston, MA, 2007. Theory, techniques, and applications.

[32] Y. Sone, K. Aoki, and T. Doi. Kinetic theory analysis of gas flows condensing on a plane condensed phase: Case of a mixture of a vapor and a noncondensable gas. Transport Theory and Statistical Physics, 21(4-6):297-328, 1992.

[33] Y. Sone, K. Aoki, S. Takata, H. Sugimoto, and A. V. Bobylev. Inappropriateness of the heat-conduction equation for description of a temperature field of a stationary gas in the continuum limit: examination 
by asymptotic analysis and numerical computation of the Boltzmann equation. Phys. Fluids, 8(2):628638, 1996.

[34] R. Strain and Y. Guo. Almost exponential decay near Maxwellian. Comm. Partial Differential Equations, 31(1-3):417-429, 2006.

[35] S. Takata and K. Aoki. The ghost effect in the continuum limit for a vapor-gas mixture around condensed phases: asymptotic analysis of the Boltzmann equation. Transport Theory Statist. Phys., 30(2-3):205237, 2001. The Sixteenth International Conference on Transport Theory, Part I (Atlanta, GA, 1999).

[36] S. Takata and F. Golse. Half-space problem of the nonlinear Boltzmann equation for weak evaporation and condensation of a binary mixture of vapors. Eur. J. Mech. B Fluids, 26(1):105-131, 2007.

[37] Y. Wang. The diffusive limit of the Vlasov-Boltzmann system for binary fluids. SIAM J. Math. Anal., 43(1):253-301, 2011.

(Carlo Bianca) Laboratoire de Physique Théorique de la Matière Condensée, Sorbonne Universités, UPMC Univ Paris 06, UMR 7600, 75252 Paris Cedex 05, France, and CNRS, UMR 7600 LPTMC, PARIS, France. E-mail address: bianca@lptmc.jussieu.it

(Christian Dogbe) Department of Mathematics, Université de Caen, LMnO, CNRS, UMR 6139, 14032 CAEn CEDEX, France. E-mail address: christian.dogbe@unicaen.fr 